\title{
Quasisymmetric Koebe uniformization
}

\author{
Sergei Merenkov and Kevin Wildrick
}

\begin{abstract}
We study a quasisymmetric version of the classical Koebe uniformization theorem in the context of Ahlfors regular metric surfaces. We provide sufficient conditions for an Ahlfors 2-regular metric space $X$ homeomorphic to a domain in the standard 2-sphere $\mathbb{S}^{2}$ to be quasisymmetrically equivalent to a circle domain in $\mathbb{S}^{2}$. We also give an example showing the sharpness of these conditions.
\end{abstract}

\section{Introduction}

Uniformization problems are amongst the oldest and most important problems in mathematical analysis. A premier example is the measurable Riemann mapping theorem, which gives a robust existence theory for quasiconformal mappings in the plane. A quasiconformal mapping between domains in a Euclidean space is a homeomorphism that sends infinitesimal balls to infinitesimal ellipsoids of uniformly bounded eccentricity. The theory of quasiconformal mappings has been one of the most fruitful in analysis, yielding applications to hyperbolic geometry, geometric group theory, complex dynamics, partial differential equations, and mathematical physics.

In the past few decades, many aspects of the theory of quasiconformal mappings have been extended to apply to abstract metric spaces. A key factor in these developments has been the realization that in metric spaces with controlled geometry, the infinitesimal condition imposed by quasiconformal mappings actually implies a stronger global condition called quasisymmetry [15]. The fact that quasisymmetric mappings are required to have good behavior at all scales makes them well suited to metric spaces that a priori have no useful infinitesimal structure.

A homeomorphism $f: X \rightarrow Y$ of metric spaces is quasisymmetric if there is a homeomorphism $\eta:[0, \infty) \rightarrow[0, \infty)$ such that if $x, y$ and $z$ are distinct points of $X$, then

$$
\frac{d_{Y}(f(x), f(y))}{d_{Y}(f(x), f(z))} \leq \eta\left(\frac{d_{X}(x, y)}{d_{X}(x, z)}\right) .
$$

Mathematics Subject Classification (2010): 30C65.

Keywords: Quasiconformal mapping, metric spaces, uniformization. 
The homeomorphism $\eta$ is called a distortion function of $f$. If we wish to emphasize that a quasisymmetric mapping has a particular distortion function $\eta$, we will call it $\eta$-quasisymmetric.

Despite the highly developed machinery for quasiconformal analysis on metric spaces, an existence theory for quasisymmetric mappings on metric spaces analogous to that of conformal mappings on Riemann surfaces has only recently been explored. The motivation for such results arises from geometric group theory [5], the dynamics of rational maps on the sphere [6], and the analysis of bi-Lipschitz mappings and rectifiable sets in Euclidean space [4].

More than a decade after foundational results of Tukia and Väisälä in dimension one [25], Bonk and Kleiner [5] gave simple sufficient conditions for a metric space to be quasisymmetrically equivalent to the standard 2 -sphere $\mathbb{S}^{2}$.

Theorem 1.1 (Bonk-Kleiner). Let $X$ be an Ahlfors 2-regular metric space homeomorphic to the sphere $\mathbb{S}^{2}$. Then $X$ is quasisymmetrically equivalent to the sphere $\mathbb{S}^{2}$ if and only if $X$ is linearly locally connected.

The condition that $X$ is linearly locally connected (LLC), which heuristically means that $X$ does not have cusps, is a quasisymmetric invariant. Ahlfors 2-regularity, which states that the two-dimensional Hausdorff measure of a ball is uniformly comparable to the square of its radius, is not. See Section 2 for precise definitions. A version of Theorem 1.1 applying to all simply connected Riemann surfaces was derived in [27], and a local version given in [28].

In this paper, we seek a version of Theorem 1.1 for a much larger class of Riemann surfaces, namely the class of domains in the sphere. Our motivation comes from analogous classical conformal uniformization theorems. The most general such result is Groetzsch's uniformization onto slit domains [11] (see Section 4). However, the geometry of a nontrivial slit domain, while harmless from the perspective of conformal mappings, is pathological with respect to quasisymmetric mappings. For this reason, it is more natural to consider uniformization onto circle domains.

A circle domain is a domain $\Omega \subseteq \mathbb{S}^{2}$ such that each component of $\mathbb{S}^{2} \backslash \Omega$ is either a round disk or a point. In 1909 [19], Koebe posed the following conjecture, known as the Kreisnormierungsproblem: every domain in the plane is conformally equivalent to a circle domain. In the 1920's [20], Koebe was able to confirm his conjecture in the finitely connected case.

Theorem 1.2 (Koebe's uniformization onto circle domains). Let $\Omega \subseteq \mathbb{C}$ be a domain with finitely many complementary components. Then $\Omega$ is conformally equivalent to a circle domain.

We first state a quasisymmetric version of Koebe's theorem, which we obtain as a consequence of our main result. Denoting the completion of a metric space $X$ by $\bar{X}$, we define the metric boundary of $X$ by $\partial X=\bar{X} \backslash X$. We say that a component of $\partial X$ is nontrivial if it contains more than one point. 
Theorem 1.3. Let $(X, d)$ be an Ahlfors 2-regular metric space that is homeomorphic to a domain in $\mathbb{S}^{2}$, and such that $\partial X$ has finitely many nontrivial components. Then $(X, d)$ is quasisymmetrically equivalent to a circle domain if and only if $(X, d)$ is linearly locally connected and the completion $\bar{X}$ is compact.

Theorem 1.3 is only quantitative in the sense that the distortion function of the quasisymmetric mapping may be chosen to depend only on the constants associated to the various conditions on $X$ and on the ratio of the diameter of $X$ to the minimum distance between components of $\partial X$. This ratio will tend to infinity as the number of components of $\partial X$ tends to infinity. It is unknown if the dependence on this ratio can be removed; see Question 12.3.

In 1993, He and Schramm confirmed Koebe's conjecture in the case of countably many complementary components [13]. In full generality the conjecture remains open. A key tool in He and Schramm's proof was transfinite induction on the rank of the boundary of a domain $\Omega$ in $\mathbb{S}^{2}$, which measures the complexity with which components of the boundary converge to one another. The rank of a collection of boundary components is defined via a canonical topology on the set of components of the boundary; see Section 3. It is shown there that if a metric space $(X, d)$ is homeomorphic to a domain $\Omega$ in $\mathbb{S}^{2}$ and is linearly locally connected, then the natural topology on the set of components of the metric boundary is homeomorphic to the natural topology on the set of boundary components of $\Omega$. This allows us to define rank as in the classical setting. We denote the topologized collection of components of $\partial X$ by $\mathcal{C}(X)$.

In the following statement, which is our main result, we consider quasisymmetric uniformization onto circle domains $\Omega$ with the property that $\mathcal{C}(\Omega)$ is uniformly relatively separated, meaning there is a uniform lower bound on the relative distance

$$
\triangle(E, F)=\frac{\operatorname{dist}(E, F)}{\min \{\operatorname{diam}(E), \operatorname{diam}(F)\}},
$$

between any pair of nontrivial boundary components. Such a circle domain is called uniformly relatively separated. This condition appears naturally in both classical quasiconformal analysis and geometric group theory. We employ annular linear local connectedness (ALLC), which is more natural than the LLC condition in this setting. Doing so removes the problem of quantitativeness present in Theorem 1.3.

Theorem 1.4. Let $(X, d)$ be a metric space, homeomorphic to a domain in $\mathbb{S}^{2}$, such that the closure of the collection of nontrivial components of $\partial X$ is countable and has finite rank. Moreover, suppose that

1. $(X, d)$ is Ahlfors 2-regular;

2. setting, for each integer $k \geq 0$,

$$
n_{k}=\sup \operatorname{card}\left\{E \in \mathcal{C}(X): E \cap B(x, r) \neq \emptyset \text { and } 2^{-k} r<\operatorname{diam} E \leq 2^{-k+1} r\right\} \text {, }
$$

where the supremum is taken over all $x \in X$ and $0<r<2 \operatorname{diam} X$, there holds

$$
\sum_{k=0}^{\infty} n_{k} 2^{-2 k}<\infty
$$


Then $(X, d)$ is quasisymmetrically equivalent to a uniformly relatively separated circle domain if and only if $X$ has the following properties:

3. the completion $\bar{X}$ is compact;

4. $(X, d)$ is annularly linearly locally connected;

5. $\mathcal{C}(X)$ is uniformly relatively separated.

Theorem 1.4 is quantitative in the sense that the distortion function of the quasisymmetric mapping may be chosen to depend only on the constants associated to the various conditions on $X$, and vice-versa.

This result is new even in the case that $(X, d)$ is assumed to be a subset of $\mathbb{S}^{2}$. The key new assumption, condition (2), controls the number of components of $\partial X$ of a given scale at any location. We note that for any Ahlfors 2-regular metric space $X$ such that $\mathcal{C}(X)$ is uniformly relatively separated, there is a constant $C \geq 1$ such that for each $k \in \mathbb{N}$, the number $n_{k}$ is bounded above by $C 2^{2 k}$. Hence, condition (2) requires a definite reduction in the number of components of $\partial X$ of a given scale and location compared to the general case.

It is of great interest to know if conditions (1) and (2) can be replaced with conditions that are quasisymmetrically invariant. By snowflaking (i.e., raising the metric to power $0<\alpha<1$ ) the sphere $\mathbb{S}^{2}=\mathbb{R}^{2} \cup\{\infty\}$ in one direction only, say, in the direction of $x$-axis, one produces a metric space homeomorphic to $\mathbb{S}^{2}$ that satisfies all the assumptions of Theorem 1.4 (and Theorem 1.1) except for Ahlfors 2 -regularity, but fails to be quasisymmetrically equivalent to $\mathbb{S}^{2}$. On the other hand, not every quasisymmetric image of $\mathbb{S}^{2}$ is Ahlfors 2-regular, as is seen by the usual snowflaking of the standard metric on $\mathbb{S}^{2}$.

Our second main result is the existence of a metric space satisfying all assumptions of Theorem 1.4, except for condition (2), that fails to quasisymmetrically embed in $\mathbb{S}^{2}$.

Theorem 1.5. There is a metric space $(X, d)$, homeomorphic to a domain in $\mathbb{S}^{2}$, with the following properties:

- $\partial X$ has rank 1 ,

- $X$ is Ahlfors 2-regular,

- the completion $\bar{X}$ is compact,

- $X$ is annularly linearly locally connected,

- the components of $\partial X$ are uniformly relatively separated,

- there is no quasisymmetric embedding of $X$ into $\mathbb{S}^{2}$.

We note that there is a locally isometric embedding of the above example into $\mathbb{S}^{2}$, and hence Theorem 1.5 highlights the fact that very strong geometric conditions, e.g., the Loewner condition, must be imposed in order to guarantee local-to-global results for quasiconformal mappings. 
Theorem 1.4 is related to work of Kapovich and Kleiner on Gromov hyperbolic groups with Sierpiński carpet boundary [18]. A conjecture of these authors states that for every Gromov hyperbolic group $G$ with boundary at infinity $\partial_{\infty} G$ homeomorphic to the Sierpiński carpet, there exists a discrete, co-compact, and isometric action of $G$ on a convex subset of hyperbolic 3-space with totally geodesic boundary. The Kapovich-Kleiner conjecture is equivalent to the following statement: if $G$ is a Gromov hyperbolic group, then $\partial_{\infty} G$ is homeomorphic to the Sierpiński carpet if and only if $\partial_{\infty} G$ is quasisymmetrically equivalent to a round Sierpiński carpet, i.e., to a subset of $\mathbb{S}^{2}$ that is homeomorphic to the Sierpiński carpent and has peripheral curves that are round circles.

Theorem 1.4 can be seen as a uniformization result for domains that might approximate a Sierpiński carpet arising as the boundary of a hyperbolic group. If $\partial_{\infty} G$ is homeomorphic to the Sierpiński carpet, then it is ALLC and the peripheral circles are uniformly separated uniform quasicirlces. Recent work of Bonk established the Kapovich-Kleiner conjecture in the case that $\partial_{\infty} G$ can be quasisymmetrically embedded in $\mathbb{S}^{2}$; see [2]. As noted by Bonk-Kleiner in [1], this is true when the Assouad dimension of $\partial_{\infty} G$ is strictly less than two, a hypothesis analgous to condition (2) in Theorem 1.4. This observation and its proof provided ideas that will be used in Section 9 .

We now outline the proof of Theorem 1.4 and the structure of the paper. In Section 3 we establish a topological characterization of the boundary components of a metric space as the space of ends of the underlying topological space, at least in the presense of some control on the geometry of the space. This allows us to develop a notion of rank, and in Section 5, a theory of crosscuts analogous to the classical theory. A key tool in this development is the following purely topological statement: every domain in $\mathbb{S}^{2}$ is homeomorphic to a domain in $\mathbb{S}^{2}$ with totally disconnected complement. This folklore theorem is proven in Section 4. In Section 6 , we use crosscuts and a classical topological recognition theorem for $\mathbb{S}^{1}$ to uniformize the boundary components of $X$. The resulting theorem, which substantially generalizes Theorem 1.3 of [27], may be of independent interest:

Theorem 1.6. Suppose that $X$ is a metric space that is homeomorphic to a domain in $\mathbb{S}^{2}$, has compact completion, and satisfies the $\lambda$-LLC condition for some $\lambda \geq 1$. Then each nontrivial component of $\partial X$ is a topological circle satisfying the $\lambda^{\prime}$-LLC condition for some $\lambda^{\prime} \geq 1$ depending only on $\lambda$. In particular, if the space $X$ is additionally assumed to be doubling, then each nontrivial component of $\partial X$ is quasisymmetrically equivalent to $\mathbb{S}^{1}$ with distortion function depending only on $\lambda$ and the doubling constant.

We emphasize that Theorem 1.6 allows for $X$ to be homeomorphic to an arbitrary domain in $\mathbb{S}^{2}$; this is the major novelty of the result and the aspect which requires the machinery of decomposition space theory.

Now, suppose that $(X, d)$ is a space satisfying the hypotheses of Theorem 1.4. By Theorem 1.6, the components of $\partial X$ are uniformly relatively separated uniform quasicircles. If there is a quasisymmetric embedding $\iota: X \hookrightarrow \mathbb{S}^{2}$, then the boundary components of the image $\iota(X)$ are again uniformly relatively separated uniform 
quasicircles. Hence, in this case, the following important uniformization result of Bonk [2] yields the conclusion of Theorem 1.4.

Theorem 1.7 (Bonk). Let $\left\{S_{i}\right\}_{i \in \mathbb{N}}$ be a collection of uniformly relatively separated uniform quasicircles in $\mathbb{S}^{2}$ that bound disjoint Jordan domains. Then there is a quasisymmetric homeomorphism $f: \mathbb{S}^{2} \rightarrow \mathbb{S}^{2}$ such that for each $i \in \mathbb{N}$, the set $f\left(S_{i}\right)$ is a round circle in $\mathbb{S}^{2}$.

In this way, the boundary uniformization given by Theorem 1.6 allows us to reduce the problem to the problem of finding a quasisymmetric embedding of $(X, d)$ into $\mathbb{S}^{2}$. We do so as follows. In Section 8 we prove general theorems implying that each nontrivial component of $\partial X$ has Assouad dimension strictly less than 2, and hence, up to a bi-Lipschitz mapping, is the boundary of a planar quasidisk. We describe a general gluing procedure in Section 9, and use it to "fill in" the nontrivial components of $\partial X$ with the corresponding planar quasidisks. The resulting space $\widehat{X}$, which contains a bi-Lipschitz copy of $X$, is again ALLC and Ahlfors 2-regular. This assertion depends crucially on condition (2) of Theorem 1.4. The assumption of finite rank allows us to reduce to the case that there are only finitely many components of $\partial X$, where we show that $\widehat{X}$ is homeomorphic to $\mathbb{S}^{2}$, and apply Theorem 1.1 to find the desired embedding. This step requires the topological uniformization of the completion of $\bar{X}$, given in Section 7 .

Acknowledgements. We wish to thank Mario Bonk, Peter Feller, Pekka Koskela, John Mackay, Daniel Meyer, Raanan Schul, and Jeremy Tyson for useful conversations and critical comments. Some of the research leading to this work took place at the following institutions: the University of Jyväskylä, the University of Illinois at Urbana-Champaign, the State University of New York at Stony Brook, and the Hausdorff Research Institute for Mathematics. We are very thankful for the hospitality of those institutions.

\section{Notation and basic results}

We are often concerned with conditions on a mapping or space that involve constants or distortion functions. These constants or distortion functions are referred to as the data of the conditions. A theorem is said to be quantitative if the data of the conclusions of theorem depend only on the data of the hypotheses. In the proof of quantitative theorems, given non-negative quantities $A$ and $B$, we will employ the notation $A \lesssim B$ if there is a quantity $C \geq 1$, depending only on the data of the conditions in the hypotheses, such that $A \leq C B$. We write $A \simeq B$ if $A \lesssim B$ and $B \lesssim A$.

\subsection{Metric spaces}

We will often denote a metric space $(X, d)$ by $X$. Given a point $x \in X$ and a number $r>0$, we define the open and closed balls centered at $x$ of radius $r$ by

$$
B_{(X, d)}(x, r)=\{y \in X: d(x, y)<r\} \quad \text { and } \quad \bar{B}_{(X, d)}(x, r)=\{y \in X: d(x, y) \leq r\} .
$$


For $0 \leq r<R$, we denote the open annulus centered at $x$ of inner radius $r$ and outer radius $R$ by

$$
A_{(X, d)}(x, r, R)=\{y \in X: r<d(x, y)<R\} .
$$

Note that when $r=0$, this corresponds to $B_{(X, d)}(x, R) \backslash\{x\}$. For $r>0$, we define a metric sphere by

$$
S_{(X, d)}(x, r)=\{y \in X: d(x, y)=r\}
$$

Where it will not cause confusion, we denote $B_{(X, d)}(x, r)$ by $B_{X}(x, r), B_{d}(x, r)$, or $B(x, r)$. A similar convention is used for all other notions which depend implicitly on the underlying metric space.

We denote the completion of a metric space $X$ by $\bar{X}$, and define the metric boundary of $X$ by $\partial X=\bar{X} \backslash X$. These notions are not to be confused with their topological counterparts.

For $\epsilon>0$, the $\epsilon$-neighborhood of a subset $E \subseteq X$ is given by

$$
\mathcal{N}_{\epsilon}(E)=\bigcup_{x \in E} B(x, \epsilon) .
$$

The diameter of $E$ is denoted by $\operatorname{diam}(E)$, and the distance between two subsets $E, F \subseteq X$ is denoted by $\operatorname{dist}(E, F)$. If at least one of $E$ and $F$ has finite diameter, then the relative distance of $E$ and $F$ is defined by

$$
\triangle(E, F)=\frac{\operatorname{dist}(E, F)}{\min \{\operatorname{diam} E, \operatorname{diam} F\}},
$$

with the convention that $\triangle(E, F)=\infty$ if at least one of $E$ and $F$ has diameter 0 .

Remark 2.1. If $f: X \rightarrow Y$ is a quasisymmetric homeomorphism of metric spaces, and $E$ and $F$ are subsets of $X$, then

$$
\triangle(f(E), f(F)) \simeq \triangle(E, F) .
$$

This is easily seen using Proposition 10.10 in [14].

Let $\mathbb{S}^{2}=\left\{(x, y, z) \in \mathbb{R}^{3}: x^{2}+y^{2}+z^{2}=1\right\}$ be the standard 2-sphere equipped with the restriction of the Euclidean metric on $\mathbb{R}^{3}$. We say that $\Omega$ is a domain in $\mathbb{S}^{2}$ if it is an open and connected subset of $\mathbb{S}^{2}$. We always consider a domain in $\mathbb{S}^{2}$ as already metrized, i.e., equipped with the restriction of the standard spherical metric.

\subsection{Dimension and measures}

A metric space $X$ is doubling if there is a constant $N \in \mathbb{N}$ such that for any $x \in X$ and $r>0$, the ball $B(x, r)$ can be covered by no more than $N$ balls of radius $r / 2$. This condition is quantitatively equivalent to the existence of constants $\alpha \geq 0$ and $C \geq 1$ such that $X$ is $(\alpha, C)$-homogeneous, meaning that for every $x \in X$, 
and $0<r \leq R$, the ball $B(x, R)$ can be covered by no more than $C(R / r)^{\alpha}$ balls of radius $r$. The infimum over all $\alpha$ such that $X$ is $(\alpha, C)$-homogeneous for some $C \geq 1$ is called the Assouad dimension of $X$. Hence, doubling metric spaces are precisely those metric spaces with finite Assouad dimension.

In a doubling metric space, some balls may have lower Assouad dimension than the entire space. To rule out this kind of non-homogeneity, one often employs a much stricter notion of finite-dimensionality. The metric space $(X, d)$ is Ahlfors $Q$-regular, $Q \geq 0$, if there is a constant $K \geq 1$ such that for all $x \in X$ and $0<r<\operatorname{diam} X$,

$$
\frac{r^{Q}}{K} \leq \mathcal{H}_{X}^{Q}(\bar{B}(x, r)) \leq K r^{Q},
$$

where $\mathcal{H}_{X}^{Q}$ denotes the $Q$-dimensional Hausdorff measure on $X$. It is quantitatively equivalent to instead require that (2.1) hold for all open balls of radius less that 2 diam $X$. The existence of any Borel regular outer measure on $X$ that satisfies (2.1) quantitatively implies that $X$ is Ahlfors $Q$-regular; see Exercise 8.11 in [14].

Remark 2.2. Suppose that $(X, d)$ is Ahlfors $Q$-regular, $Q \geq 0$. Given $S \subseteq \partial X$, the space $(X \cup S, d)$ is again Ahlfors $Q$-regular, quantitatively. This is proven as in Lemma 2.11 of [27].

\subsection{Connectivity conditions}

Here we describe various conditions that control the existence of "cusps" in a metric space by means of connectivity. The basic concept of such conditions arose from the theory of quasiconformal mappings in the plane, where they play an important role as invariants.

Let $\lambda \geq 1$. A metric space $(X, d)$ is $\lambda$-linearly locally connected ( $\lambda$-LLC) if for all $a \in X$ and $r>0$, the following two conditions are satisfied:

(i) for each pair of distinct points $x, y \in B(a, r)$, there is a continuum $E \subseteq$ $B(a, \lambda r)$ such that $x, y \in E$,

(ii) for each pair of distinct points $x, y \in X \backslash B(a, r)$, there is a continuum $E \subseteq$ $X \backslash B(a, r / \lambda)$ such that $x, y \in E$.

Individually, conditions (i) and (ii) are referred to as the $\lambda$-LLC 1 and $\lambda$-LLC 2 conditions, respectively.

The LLC condition extends in a particularly nice way to the completion of a metric space. We say that a metric space $(X, d)$ is $\lambda$-LLC if for all $a \in \bar{X}$ and $r>0$ the following conditions are satisfied:

(i) For each pair of distinct points $x, y \in B_{\bar{X}}(a, r)$, there is an embedding $\gamma:[0,1] \rightarrow \bar{X}$ such that $\gamma(0)=x, \gamma(1)=y,\left.\gamma\right|_{(0,1)} \subseteq X$, and $\operatorname{im} \gamma \subseteq$ $B_{\bar{X}}(a, \lambda r)$,

(ii) For each pair of distinct points $x, y \in \bar{X} \backslash B_{\bar{X}}(a, r)$, there is an embedding $\gamma:[0,1] \rightarrow \bar{X}$ such that $\gamma(0)=x, \gamma(1)=y,\left.\gamma\right|_{(0,1)} \subseteq X$, and $\operatorname{im} \gamma \subseteq$ $\bar{X} \backslash B_{\bar{X}}(a, r / \lambda)$. 
Individually, conditions (i) and (ii) are referred to as the $\lambda$ - $\widetilde{\mathrm{LLC}}_{1}$ and $\lambda$-LLC $\widetilde{\mathrm{LL}}_{2}$ conditions, respectively.

If a metric space $X$ is $\lambda$-LLC, then it is also $\lambda$-LLC. The next proposition states that the two conditions are quantitatively equivalent for the spaces considered in this paper.

Proposition 2.3. Let $i \in\{1,2\}$, and let $(X, d)$ be a locally compact and locally path-connected metric space that satisfies the $\lambda$-LLC $\mathrm{L}_{i}$ condition. Then $X$ is $\lambda^{\prime}-\widetilde{\mathrm{LLC}}_{i}$, where $\lambda^{\prime}$ depends only on $\lambda$.

Proof. The key ingredient is the following statement: If $U \subseteq X$ is an open subset of $X$, and $E \subseteq U$ is a continuum, then any pair of points $x, y \in E$ are contained in an $\operatorname{arc}$ in $U$. The details are straightforward and left to the reader.

Let $\lambda \geq 1$. A metric space $\left(X, d_{X}\right)$ is $\lambda$-annularly linearly locally connected ( $\lambda$-ALLC) if for all points $a \in X$ and all $0 \leq r<R$, each pair of distinct points in the annulus $A(a, r, R)$ is contained in a continuum in the annulus $A(a, r / \lambda, \lambda R)$.

The ALLC condition forbids local cut-points in addition to ruling out cusps. For example, the standard circle $\mathbb{S}^{1}$ is LLC but not ALLC. In our setting, the ALLC condition is a more natural assumption, and is in some cases equivalent to the LLC condition.

We omit the proofs of the following three statements. The first is based on decomposing an arbitrary annulus into dyadic annuli. The second uses the fact that in a connected space, any distinct pair of points is contained in an annulus around some third point. The third states that the ALLC condition extends to the boundary as in Proposition 2.3 and is proven similarly.

Lemma 2.4. Let $\lambda \geq 1$. Suppose that a connected metric space $(X, d)$ satisfies the condition that for all points $a \in X$ and all $r>0$, each pair of distinct points in the annulus $A(a, r, 2 r)$ is contained in a continuum in the annulus $A(a, r / \lambda, 2 \lambda r)$. Then $X$ satisfies the $\lambda$-ALLC condition.

Lemma 2.5. Suppose that $(X, d)$ is a connected metric space that satisfies the $\lambda$-ALLC condition. Then $(X, d)$ satisfies the $2 \lambda$-LLC condition.

Proposition 2.6. Suppose that $(X, d)$ is a locally compact and locally path-connected metric space that satisfies the $\lambda$-ALLC condition. Then there is a quantity $\lambda^{\prime} \geq 1$, depending only on $\lambda$, such that for all $a \in \bar{X}$ and $0 \leq r<R$, and for each pair of distinct points $x, y \in A_{\bar{X}}(a, r, R)$, there is an embedding $\gamma:[0,1] \rightarrow \bar{X}$ such that $\gamma(0)=x, \gamma(1)=y,\left.\gamma\right|_{(0,1)} \subseteq X$, and

$$
\operatorname{im} \gamma \subseteq A_{\bar{X}}\left(a, r / \lambda^{\prime}, \lambda^{\prime} R\right)
$$

There is a close connection between the ALLC condition and the uniform relative separation of the components of the boundary of a given metric space. The following statement addresses only circle domains, but a more general result is probably valid. We postpone the proof until Section 4. 
Proposition 2.7. Let $\Omega$ be a circle domain. Then $\Omega$ satisfies the ALLC condition if and only if the components of $\partial \Omega$ are uniformly relatively separated, quantitatively.

In the case of metric spaces that are homeomorphic to domains in $\mathbb{S}^{2}$ with finitely many boundary components, the LLC-condition may be upgraded to the ALLC-condition. Again, we postpone the proof until Section 4.

Proposition 2.8. Let $(X, d)$ be a metric space homeomorphic to a domain in $\mathbb{S}^{2}$, and assume that the boundary $\partial X$ has finitely many components. If $(X, d)$ is $\lambda$-LLC, $\lambda \geq 1$, then it is $\Lambda$-ALLC, where $\Lambda$ depends on $\lambda$ and the ratio of the diameter of $X$ to the minimum distance between components of $\partial X$.

\section{The space of boundary components of a metric space}

In this section we assume that $(X, d)$ is a connected, locally compact metric space with the additional property that the completion $\bar{X}$ is compact. Note that as $X$ is locally compact, it is an open subset of $\bar{X}$. Hence $\partial X$ is closed in $\bar{X}$ and therefore compact.

\subsection{Boundary components and ends}

Of course, the topological type of $\partial X$ depends on the specific metric $d$. However, the goal of this section is to show that under a simple geometric condition, the collection $\mathcal{C}(X)$ of components of $\partial X$ depends only on the topological type of $X$.

Remark 3.1. This goal cannot be realized without some control on the geometry of $X$. The following example was pointed out to us by Daniel Meyer. Let $(r, \theta, z)$ denote cylindrical coordinates on $\mathbb{R}^{3}$, and set

$$
X=\left\{(r, \theta, z):(r-1)^{2}+z^{2}=1\right\} \backslash\{(0,0,0)\} .
$$

Heuristically, $X$ is a sphere in $\mathbb{R}^{3}$ with its poles pinched together. Equipped with the standard metric inherited from $\mathbb{R}^{3}$, the space $X$ is homeomorphic to a punctured disk, and hence has two ends. However, the metric boundary of $X$ consists only of the point $\{(0,0,0)\}$.

We define an equivalence relation $\sim$ on $\partial X$ by declaring that $x \sim y$ if and only if $x$ and $y$ are contained in the same component of $\partial X$. Then there is a bijection between $\mathcal{C}(X)$ and the quotient $\partial X / \sim$, and hence we may endow $\mathcal{C}(X)$ with the quotient topology. Since $\partial X$ is compact, the space $\mathcal{C}(X)$ is compact as well. Given a compact set $K \subseteq X$ and a component $U$ of $X \backslash K$, denote

$$
\mathcal{C}(K, U)=\{E \subseteq \mathcal{C}(X): E \subseteq(\partial U \cap \partial X)\} .
$$

Let $\mathcal{U}(X)$ denote the collection of sequences $\left\{x_{i}\right\} \subseteq X$ with the property that for every compact set $K \subseteq X$, there is a number $N \in \mathbb{N}$ and a connected subset $U$ of $X \backslash K$ such that

$$
\left\{x_{i}\right\}_{i \geq N} \subseteq U .
$$


Define an equivalence relation $\stackrel{e}{\sim}$ on $\mathcal{U}(X)$ by $\left\{x_{i}\right\} \stackrel{e}{\sim}\left\{y_{i}\right\}$ if and only if the sequence $\left\{x_{1}, y_{1}, x_{2}, y_{2}, \ldots\right\}$ is in $\mathcal{U}(X)$. An equivalence class $E$ defined by $\stackrel{e}{\sim}$ is called an end of $X$, and we denote the collection of ends of $X$ by $\mathcal{E}(X)$.

Given a compact subset $K \subseteq X$ and a component $U$ of $X \backslash K$, define

$$
\mathcal{E}(K, U)=\left\{\left[\left\{x_{i}\right\}\right]:\left\{x_{i}\right\} \in \mathcal{U}(X) \text { and } \exists N \in \mathbb{N} \text { such that }\left\{x_{i}\right\}_{i \geq N} \subseteq U\right\} .
$$

That this set is well defined follows from the definition of the equivalence relation on $\mathcal{U}(X)$. Let $\mathcal{B}$ be the collection of all such sets.

Proposition 3.2. The collection $\mathcal{B}$ generates a unique topology on $\mathcal{E}(X)$ such that every open set is a union of sets in $\mathcal{B}$.

Proof. We employ the standard criteria for proving generation (see Section 13 in [24]). As $X$ is connected, taking $K=\emptyset$ shows that $\mathcal{B}$ contains $\mathcal{E}(X)$. Thus it suffices to show that given compact subsets $K_{1}$ and $K_{2}$ of $X$ and components $U_{1}$ and $U_{2}$ of $X \backslash K_{1}$ and $X \backslash K_{2}$ respectively, and given an end

$$
E \in \mathcal{E}\left(K_{1}, U_{1}\right) \cap \mathcal{E}\left(K_{2}, U_{2}\right),
$$

there is a compact set $K$ and a component $U$ of $X \backslash K$ such that

$$
E \in \mathcal{E}(K, U) \subseteq\left(\mathcal{E}\left(K_{1}, U_{1}\right) \cap \mathcal{E}\left(K_{2}, U_{2}\right)\right)
$$

Let $\left\{x_{i}\right\} \in \mathcal{U}(X)$ represent the end $E$. By definition of $\mathcal{U}(X)$, there is a component $U$ of $X \backslash K$, where $K=K_{1} \cup K_{2}$, such that $\left\{x_{i}\right\}_{i \geq N} \subseteq U$ for some $N \in \mathbb{N}$. This implies that $E \in \mathcal{E}(K, U)$. By assumption, there is a number $M \in \mathbb{N}$ such that $\left\{x_{i}\right\}_{i \geq M}$ is contained in $U_{1} \cap U_{2}$. Since $U$ is a connected subset of $X \backslash K_{1}$ and $X \backslash K_{2}$, it follows that $U \subseteq U_{1} \cap U_{2}$. This implies (3.1).

We set the topology on $\mathcal{E}(X)$ to be that given by Proposition 3.2. It follows quickly from the definitions that $\mathcal{E}(X)$ is a Hausdorff space.

Remark 3.3. As ends are defined in purely topological terms, a homeomorphism $h: X \rightarrow Y$ to some other topological space $Y$ induces a homeomorphism $\phi: \mathcal{E}(X) \rightarrow \mathcal{E}(Y)$. This homeomorphism is natural in the sense that a sequence $\left\{x_{i}\right\} \in \mathcal{U}(X)$ represents the end $E \in \mathcal{E}(X)$ if and only $\left\{h\left(x_{i}\right)\right\} \in \mathcal{U}(Y)$ represents the end $\phi(E) \in \mathcal{E}(Y)$.

To relate the ends of a metric space to the components of its metric boundary, we need an elementary result regarding connectivity. Let $\epsilon>0$ and let $x$ and $y$ be points in a metric space $Z$. An $\epsilon$-chain connecting $x$ to $y$ in $Z$ is a sequence $x=$ $z_{0}, z_{1}, \ldots, z_{n}=y$ of points in $Z$ such that $d\left(z_{j}, z_{j+1}\right) \leq \epsilon$ for each $j=0, \ldots, n-1$. In compact spaces, the existence of arbitrarily fine chains can detect connectedness. We leave the proof of the following statement to that effect to the reader.

Lemma 3.4. Let $x$ and $y$ be points in a metric space $Z$. If $x$ and $y$ lie in the same component of $Z$, then for every $\epsilon>0$ there is an $\epsilon$-chain in $Z$ connecting $x$ to $y$. The converse statement holds if $Z$ is compact. 
An end always defines a unique boundary component.

Proposition 3.5. If $\left\{x_{i}\right\}$ and $\left\{y_{i}\right\}$ are Cauchy sequences representing the same end of $X$, then they represent points in the same component of $\partial X$.

Proof. Denote by $x$ and $y$ the points in $\partial X$ defined by $\left\{x_{i}\right\}$ and $\left\{y_{i}\right\}$, respectively. By Lemma 3.4, in order to show that $x$ and $y$ are contained in a single component of $\partial X$, it suffices to find an $\epsilon$-chain in $\partial X$ connecting $x$ to $y$ for every $\epsilon>0$. To this end, fix $\epsilon>0$. Let $K$ be the compact subset of $X$ defined by

$$
K=X \backslash \mathcal{N}_{\bar{X}}(\partial X, \epsilon / 3) .
$$

By assumption, we may find $N \in \mathbb{N}$ so large that $x_{N}$ and $y_{N}$ lie in a connected subset of $X \backslash K$, and that $d\left(x, x_{N}\right)$ and $d\left(y, y_{N}\right)$ are both less than $\epsilon / 3$. By Lemma 3.4, we may find an $\epsilon / 3$-chain $x_{N}=z_{0}, \ldots, z_{n}=y_{N}$ in $X \backslash K$. By (3.2), for each $j=1, \ldots, n-1$ we may find a point $z_{j}^{\prime} \in \partial X$ such that $d\left(z_{j}, z_{j}^{\prime}\right)<\epsilon / 3$. The triangle inequality now implies that $x, z_{1}^{\prime}, \ldots, z_{n-1}^{\prime}, y$ is an $\epsilon$-chain in $\partial X$, as required.

Remark 3.6. Proposition 3.5 allows us to define a map $\Phi: \mathcal{E}(X) \rightarrow \mathcal{C}(X)$ as follows. Let $E \in \mathcal{E}(X)$ and let $\left\{x_{i}\right\}_{i \in \mathbb{N}} \in \mathcal{U}(X)$ be a sequence representing $E$. As $\bar{X}$ is compact, we may find a Cauchy subsequence $\left\{x_{i_{j}}\right\}$ of $\left\{x_{i}\right\}$ that represents a point in some boundary component $E^{\prime} \in \mathcal{C}(X)$. Set $\Phi(E)=E^{\prime}$. This is well defined by Proposition 3.5.

We now consider when a boundary component $E \in \mathcal{C}(X)$ corresponds to an end. For subspaces of $\mathbb{S}^{2}$, this is always the case. The key tool in the proof of this is the following purely topological fact, which is mentioned in the proof of Lemma 2.5 in $[5]$.

Proposition 3.7. Each domain $\Omega$ in $\mathbb{S}^{2}$ may be written as a union of open and connected subsets $\Omega_{1} \subseteq \Omega_{2} \subseteq \cdots$ of $\Omega$ such that for each $i \in \mathbb{N}$, the closure of $\Omega_{i}$ is a compact subset of $\Omega$ and $\partial \Omega_{i}$ is a finite collection of pairwise disjoint Jordan curves.

Proposition 3.8. Let $\Omega$ be a domain in $\mathbb{S}^{2}$. If $x$ and $y$ are points in the same component of $\partial \Omega$, then any Cauchy sequences representing $x$ and $y$ are in $\mathcal{U}(\Omega)$ and represent the same end of $\Omega$.

Proof. The metric boundary $\partial \Omega$ coincides with the usual topological boundary of $\Omega$ in $\mathbb{S}^{2}$. Let $\left\{\Omega_{i}\right\}$ denote the exhaustion of $\Omega$ provided by Proposition 3.7. Since $x$ and $y$ are in the same component of $\partial \Omega$, for each $i \in \mathbb{N}$ they belong to a single simply connected component $U_{i}$ of $\mathbb{S}^{2} \backslash \Omega_{i}$. Let $\left\{x_{i}\right\}$ and $\left\{y_{i}\right\}$ be Cauchy sequences representing $x$ and $y$ respectively.

Suppose that $K$ is a compact subset of $\Omega$. We may find $i_{0} \in \mathbb{N}$ so large that $\Omega_{i_{0}} \supseteq K$. Then $U_{i_{0}} \cap \Omega$ does not intersect $K$. Moreover, there is a number $N \in \mathbb{N}$ such that

$$
\left(\left\{x_{i}\right\}_{i \geq N} \cup\left\{y_{i}\right\}_{i \geq N}\right) \subseteq U_{i_{0}} \cap \Omega
$$


It now suffices to show that $U_{i_{0}} \cap \Omega$ is connected. Let $a$ and $b$ be points in $U_{i_{0}} \cap \Omega$. We may find $j \geq i_{0}$ so large that $a$ and $b$ are contained in $\Omega_{j}$. The set $U_{i_{0}} \cap \Omega_{j}$ is a simply connected domain with finitely many disjoint closed topological disks removed from its interior, and is therefore path-connected. Thus $a$ and $b$ may be connected by a path inside $U_{i_{0}} \cap \Omega_{j}$, and hence inside $U_{i_{0}} \cap \Omega$.

The proof given above does not even pass to metric spaces that are merely homeomorphic to a domain in $\mathbb{S}^{2}$, as it need not be the case that the completion of such a space embeds topologically in $\mathbb{S}^{2}$ (recall Remark 3.1). However, under an additional assumption controlling the geometry of $X$, we can give a different proof.

Proposition 3.9. Suppose that $X$ satisfies the $\mathrm{LLC}_{1}$ condition. If $x$ and $y$ are points in the same component of $\partial X$, then any Cauchy sequences representing $x$ and $y$ are in $\mathcal{U}(X)$ and represent the same end of $X$.

Lemma 3.10. Suppose that $X$ satisfies the $\lambda-\mathrm{LLC}_{1}$ condition for some $\lambda \geq 1$. Let $E$ be a connected subset of $\partial X$ and let $\epsilon>0$. Then $\mathcal{N}_{\bar{X}}(E, \epsilon) \cap X$ is contained in a connected subset of $\mathcal{N}_{\bar{X}}(E, 3 \lambda \epsilon) \cap X$.

Proof. It suffices to show that if $x$ and $y$ are points in $\mathcal{N}_{\bar{X}}(E, \epsilon) \cap X$, then there is a continuum containing $x$ to $y$ inside of $\mathcal{N}_{\bar{X}}(E, 3 \lambda \epsilon) \cap X$. Let $x^{\prime}$ and $y^{\prime}$ be points in $E$ such that $d\left(x, x^{\prime}\right)<\epsilon$ and $d\left(y, y^{\prime}\right)<\epsilon$. By Lemma 3.4, there is an $\epsilon$-chain $x^{\prime}=z_{0}^{\prime}, \ldots, z_{n}^{\prime}=y^{\prime}$ in $E$. For each $j=1, \ldots, n-1$, find a point $z_{j} \in X$ such that $d\left(z_{j}, z_{j}^{\prime}\right)<\epsilon$. The triangle inequality implies that for $j=0, \ldots, n-1$,

$$
z_{j+1} \in B_{X}\left(z_{j}, 3 \epsilon\right) .
$$

Repeatedly applying the $\lambda-\mathrm{LLC}_{1}$ condition and concatenating now yields the desired result.

Proof of Proposition 3.9. Let $x$ and $y$ be points in a connected subset $E$ of $\partial X$, and let $\left\{x_{i}\right\}$ and $\left\{y_{i}\right\}$ be Cauchy sequences in $X$ corresponding to $x$ and $y$, respectively. Let $K$ be a compact subset of $X$. As $\bar{X}$ is compact, we may find $\epsilon>0$ such that $\operatorname{dist}(E, K)>3 \lambda \epsilon$. Let $N \in \mathbb{N}$ be so large that

$$
\left\{x_{i}\right\}_{i \geq N} \cup\left\{y_{i}\right\}_{i \geq N} \subseteq \mathcal{N}_{\bar{X}}(E, \epsilon) \cap X .
$$

Lemma 3.10 now implies the desired results.

The following statement is the main result of this section. In the case that $X$ is a domain in $\mathbb{S}^{2}$, the statement is mentioned in [13]. We recall that $X$ is assumed to be a connected and locally compact metric space with the additional assumption that $\bar{X}$ is compact.

Theorem 3.11. Suppose that $X$ is either a domain in $\mathbb{S}^{2}$ or satisfies the $\mathrm{LLC}_{1}$ condition. Then the map $\Phi: \mathcal{E}(X) \rightarrow \mathcal{C}(X)$ defined in Remark 3.6 is a homeomorphism that is natural in the sense that a Cauchy sequence $\left\{x_{i}\right\} \in \mathcal{U}(X)$ represents the end $E \in \mathcal{E}(X)$ if and only if it represents a point on the boundary component $\phi(E) \in \mathcal{C}(X)$. 
In the proof of the following lemma, we consider only the case that $X$ satisfies the $\mathrm{LLC}_{1}$ condition. If $X$ is a domain in $\mathbb{S}^{2}$, a proof is easily constructed using Proposition 3.7.

Lemma 3.12. Suppose that $X$ is a domain in $\mathbb{S}^{2}$ or satisfies the $\mathrm{LLC}_{1}$ condition. Let $K$ be a compact subset of $X$ and let $U$ be a component of $X \backslash K$. Then the following statements hold:

(i) for each $x \in \bar{U} \cap \partial X$, there is a number $\delta>0$ such that $B_{\bar{X}}(x, \delta) \cap X \subseteq U$;

(ii) if $E \in \mathcal{C}(X)$ intersects $\partial U \cap \partial X$, then $E \in \mathcal{C}(K, U)$;

(iii) the set $\mathcal{C}(K, U)$ is open in $\mathcal{C}(X)$.

Proof. We assume that $X$ satisfies the $\lambda$-LLC 1 condition for some $\lambda \geq 1$.

Suppose that statement (i) is not true. Then for all sufficiently small $\delta>0$ we may find points $a, b \in B_{\bar{X}}(x, \delta) \cap X$ such that $a \in U$ and $b$ is in some other component of $X \backslash K$. Using the $\lambda$-LLC $\mathrm{LL}_{1}$ condition to connect $a$ to $b$ inside of $B_{X}(a, 2 \lambda \delta)$ produces a point of $K$ in the ball $B_{\bar{X}}(x, 3 \lambda \delta)$. Letting $\delta$ tend to 0 produces a contradiction with the assumption that $K$ is a compact subset of $X$.

Statement (i) implies that the collection

$$
\{E \cap \partial V \cap \partial X: V \text { is a component of } X \backslash K\}
$$

consists of pairwise disjoint open subsets of $E$. Hence the connectedness of $E$ proves statement (ii).

Now, recall that $\mathcal{C}(X)$ is endowed with the quotient topology. Hence, by statement (ii), in order to show that $\mathcal{C}(K, U)$ is open in $\mathcal{C}(X)$, it suffices to show that $\cup_{E \in \mathcal{C}(K, U)} E$ is open in $\partial X$. This follows from statements (i) and (ii).

Proof of Theorem 3.11. Proposition 3.8 or 3.9 shows that $\Phi$ is injective. Given $E^{\prime} \in \mathcal{C}(X)$ and a Cauchy sequence $\left\{x_{i}\right\}_{i \in \mathbb{N}}$ representing a point in $E^{\prime}$, Proposition 3.8 or 3.9 also state that $\left\{x_{i}\right\}_{i \in \mathbb{N}}$ is in $\mathcal{U}(X)$ and hence represents an end $E \in \mathcal{E}(X)$. By definition, this implies that $\Phi(E)=E^{\prime}$, and so $\Phi$ is surjective.

We now check that the bijection $\Phi$ is a homeomorphism. Since $\mathcal{C}(X)$ is compact and $\mathcal{E}(X)$ is Hausdorff, this is true if $\Phi^{-1}$ is continuous. Hence, by Lemma 3.12 (iii) and the definition of the topology on $\mathcal{E}(X)$, it suffices to show that for any compact set $K \subseteq X$ and any component $U$ of $X \backslash K$,

$$
\Phi(\mathcal{E}(K, U))=\mathcal{C}(K, U)
$$

Let $E$ be an end in $\mathcal{E}(K, U)$. By definition, the limit of any Cauchy sequence representing $E$ lies in $\partial U \cap \partial X$. Hence, $\Phi(E)$ intersects $\partial U \cap \partial X$, and so Lemma 3.12 (ii) shows that $\Phi(E) \in \mathcal{C}(K, U)$. Now, let $E^{\prime} \in \mathcal{C}(K, U)$ and choose a Cauchy sequence $\left\{x_{i}\right\}_{i \in \mathbb{N}}$ representing a point in $E^{\prime}$. Lemma 3.12 (i) implies that there is $N \in \mathbb{N}$ such that $\left\{x_{i}\right\}_{i \geq N}$ is contained in $U$. Again, Proposition 3.8 or 3.9 states that $\left\{x_{i}\right\}$ is in $\mathcal{U}(X)$ and hence represents an end $E$. Thus, by definition, $E \in \mathcal{E}(K, U)$ and $\Phi(E)=E^{\prime}$. 


\subsection{Rank}

We briefly recall the notion of rank as discussed in [13]. Let $\mathcal{T}$ be a countable, compact, and Hausdorff topological space. Set $\mathcal{T}^{0}=\mathcal{T}$, and for each $n \geq 1$, set $\mathcal{T}^{n}$, to be the set of non-isolated points of $\mathcal{T}^{n-1}$, and endow $\mathcal{T}^{n}$ with the subspace topology. This process can be continued using transfinite induction to define $\mathcal{T}^{\alpha}$ for each ordinal $\alpha$, though we will not need this. For each ordinal $\alpha$, the space $\mathcal{T}^{\alpha}$ is again countable, compact, and Hausdorff. By the Baire category theorem, there is a unique ordinal $\alpha$ such that $\mathcal{T}^{\alpha}$ is finite and nonempty; this ordinal is defined to be the rank of $\mathcal{T}$.

Let $(X, d)$ be a metric space that is either a domain in $\mathbb{S}^{2}$ or satisfies the $\mathrm{LLC}_{1}$ - condition. By Theorem 3.11, the space of boundary components $\mathcal{C}(X)$ is homeomorphic to the space of ends $\mathcal{E}(X)$. As mentioned above, the former is clearly compact and the latter clearly Hausdorff, hence both are compact Hausdorff spaces. Hence, if $\mathcal{S}$ is a closed and countable subset of $\mathcal{C}(X)$, the rank of $\mathcal{S}$ is defined.

\section{Domains with totally disconnected complement}

The simplest possible structure of a boundary component is that it consists of a single point. The aim of this section is to show that if we are only concerned with the topological properties, we may always assume this is the case.

Proposition 4.1. Every domain in $\mathbb{S}^{2}$ is homeomorphic to a domain in $\mathbb{S}^{2}$ that has totally disconnected complement.

To prove Proposition 4.1, we employ the theory of decomposition spaces [8]. A decomposition of a topological space $S$ is simply a partition $G$ of $S$. The nondegenerate elements of a decomposition are those elements of the partition that contain at least two points. The decomposition space $S / G$ associated to a decomposition $G$ of a topological space $S$ is the topological quotient of $S$ obtained by, for each $g \in G$, identifying the points of $g$. A decomposition $G$ is an upper semicontinuous decomposition if each element is compact, and given any $g \in G$ and any open set $U \subseteq S$ containing $g$, there is another open set $V$ containing $g$ with the property that if $g^{\prime} \in G$ intersects $V$, then $g^{\prime} \subseteq U$. If $G$ is an upper semicontinuous decomposition of a separable metric space $S$, then $S / G$ is a separable and metrizable space (see Proposition I.2.2 in [8]).

We will use one powerful theorem from classical decomposition space theory. It identifies decompositions of $\mathbb{S}^{2}$ that are homeomorphic to $\mathbb{S}^{2}$ itself [23].

Theorem 4.2 (Moore). Suppose that $G$ is an upper semicontinuous decomposition of $\mathbb{S}^{2}$ that has at least two elements and has the property that for each $g \in G$, both $g$ and $\mathbb{S}^{2} \backslash g$ are connected. Then $S / G$ is homeomorphic to $\mathbb{S}^{2}$.

We also employ a powerful theorem, due to Grötzsch [11], of classical complex analysis. It states that any domain in $\mathbb{S}^{2}$ can be mapped conformally (and hence 
homeomorphically) to a slit domain, i.e., to a domain in $\mathbb{S}^{2}$ that is either complete, or whose complementary components are points or compact horizontal line segments in $\mathbb{R}^{2}$. See [10], V.2.

Theorem 4.3. Any domain in $\mathbb{S}^{2}$ is conformally equivalent to a slit domain.

Lemma 4.4. If $\Omega \subseteq \mathbb{S}^{2}$ is a slit domain, then the components of $\mathbb{R}^{2} \backslash \Omega$ containing at least two points form the non-degenerate elements of an upper semicontinuous decomposition of $\mathbb{S}^{2}$.

Proof. Let $G$ denote the decomposition of $\mathbb{S}^{2}$ whose non-degenerate elements are those components of $\mathbb{R}^{2} \backslash \Omega$ that contain at least two points. Let $g \in G$, and let $U \subseteq \mathbb{S}^{2}$ be an open set containing $g$. Without loss of generality we may assume that $g=[0,1] \times\{0\}$ and that $U$ is an open and bounded subset of $\mathbb{R}^{2}$ containing $g$.

Set

$l=\max \left\{x:(x, 0) \in \mathbb{R}^{2} \backslash U\right.$ and $\left.x<0\right\}$ and $r=\min \left\{x:(x, 0) \in \mathbb{R}^{2} \backslash U\right.$ and $\left.x>1\right\}$.

Since $\Omega \cap U$ is open and $g$ is a compact and connected subset of $U$, there are closed, non-degenerate intervals $L, R \subseteq \mathbb{R}$ such that

$$
(L \times\{0\}) \subseteq((l, 0) \times\{0\}) \cap \Omega \cap U \text { and }(R \times\{0\}) \subseteq((1, r) \times\{0\}) \cap \Omega \cap U .
$$

If $G$ is not upper semicontinuous, then for every $n \in \mathbb{N}$, we may find some horizontal line segment $g_{n} \in G$ and points $\left(x_{n}, y_{n}\right),\left(x_{n}^{\prime}, y_{n}\right) \in \mathbb{R}^{2}$ such that

$$
\left(x_{n}, y_{n}\right) \in g_{n} \cap \mathcal{N}(g, 1 / n) \text { and }\left(x_{n}^{\prime}, y_{n}\right) \in g_{n} \cap\left(\mathbb{S}^{2} \backslash U\right) .
$$

After passing to a subsequence, we may assume that $\left(x_{n}, y_{n}\right)$ tends to a point of $g$. Moreover, passing to another subsequence if needed, we may assume that either

$$
\limsup _{n \rightarrow \infty} x_{n}^{\prime} \leq l \quad \text { or } \quad \liminf _{n \rightarrow \infty} x_{n}^{\prime} \geq r .
$$

We consider the latter case; a similar argument applies in the former. For sufficiently large $n$, the point $x_{n}^{\prime}$ is greater than any point in $R$, while $x_{n}$ is less than any point in $R$. By the connectedness of $g_{n}$, we conclude that there is a point $\left(z_{n}, y_{n}\right) \in g_{n}$ with $z_{n} \in R$. After passing to yet another subsequence, we may assume that $\left(z_{n}, y_{n}\right)$ converges to a point in $R \times\{0\}$. This is a contradiction as $\Omega$ is open. See Figure 1.

Proof of Proposition 4.1. Let $\Omega$ be a domain in $\mathbb{S}^{2}$. By Theorem 4.3, there is a homeomorpism $h_{0}: \Omega \rightarrow \Omega_{0}$, where $\Omega_{0}$ is a slit domain. By Lemma 4.4, the components of $\mathbb{S}^{2} \backslash \Omega_{0}$ with at least two points form the non-degenerate elements of an upper semicontinuous decomposition $G$ of $\mathbb{S}^{2}$. As each element of $G$ is either a point or a compact line segment in $\mathbb{R}^{2}$, the hypotheses of Theorem 4.2 are satisfied. Thus there is a homeomorphism $h_{1}: \mathbb{S}^{2} / G \rightarrow \mathbb{S}^{2}$. Let $\pi: \mathbb{S}^{2} \rightarrow \mathbb{S}^{2} / G$ denote the standard projection map. By definition $\left.\pi\right|_{\Omega_{0}}$ is a homeomorphism and $\pi\left(\mathbb{S}^{2} \backslash \Omega_{0}\right)$ is totally disconnected. Thus $h_{1} \circ \pi \circ h_{0}: \Omega \rightarrow \mathbb{S}^{2}$ is a homeomorphism, and the image of $\Omega$ under this map has totally disconnected complement. 


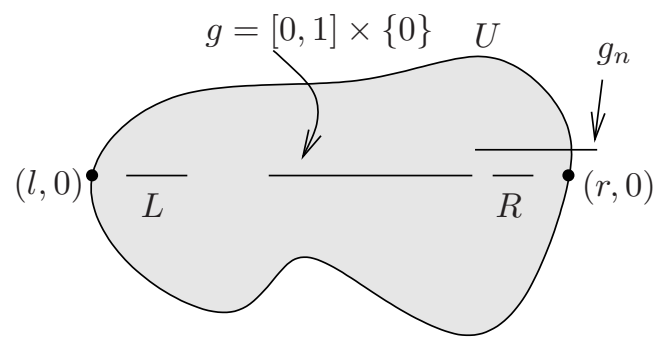

FIGURE 1. If upper semi-continuity fails.

The ends of a domain in $\mathbb{S}^{2}$ with totally disconnected complement are particularly easy to understand: they are in bijection with the points of the complement, which are precisely the boundary components.

Proposition 4.5. Suppose $\Omega$ is a domain in $\mathbb{S}^{2}$ with totally disconnected complement. Then there is a homeomorphism $\phi: \mathcal{E}(\Omega) \rightarrow \partial \Omega$ with the property that a sequence $\left\{x_{n}\right\} \in \mathcal{U}(\Omega)$ represents the end $E \in \mathcal{E}(\Omega)$ if and only if it converges to $\phi(E)$.

Proof. Since a totally disconnected subset of $\mathbb{S}^{2}$ cannot have interior, we see that $\mathbb{S}^{2} \backslash \Omega=\partial \Omega$. Moreover, it is clear that $\mathcal{C}(\Omega)$ and $\partial \Omega$ are naturally homeomorphic. Hence Theorem 3.11 provides the desired homeomorphism.

The following statement transfers the work of this section to the general setting. For the remainder of this section, we assume that $(X, d)$ is a metric space that has compact completion and is homeomorphic to a domain in $\mathbb{S}^{2}$.

Corollary 4.6. Suppose that $X$ satisfies the $\mathrm{LLC}_{1}$ condition or is a domain in $\mathbb{S}^{2}$. Then there is a continuous surjection $h: \bar{X} \rightarrow \mathbb{S}^{2}$ such that $\left.h\right|_{X}$ is a homeomorphism onto a domain $\Omega$ with totally disconnected complement. Moreover, the map $h$ is constant on each boundary component $E \in \mathcal{C}(X)$, and for any $\epsilon>0$, there is $\epsilon^{\prime}>0$ such that

$$
h^{-1}\left(B_{\mathbb{S}^{2}}\left(h(E), \epsilon^{\prime}\right)\right) \subseteq \mathcal{N}_{\bar{X}}(E, \epsilon) .
$$

Finally, $h$ induces a homeomorphism from $\mathcal{C}(X)$ to $\partial \Omega$.

Proof. We address only the case that $X$ satisfies the $\mathrm{LLC}_{1}$ condition. We have assumed that $X$ is homeomorphic to a domain in $\mathbb{S}^{2}$. Hence by Proposition 4.1, there is a homeomorphism $h: X \rightarrow \Omega$, where $\Omega \subseteq \mathbb{S}^{2}$ is a domain with totally disconnected complement.

By Theorem 3.11 and Remark 3.3, there is a homeomorphism $\phi_{0}: \mathcal{C}(X) \rightarrow \mathcal{E}(\Omega)$ with the property that a Cauchy sequence $\left\{x_{i}\right\} \in \mathcal{U}(X)$ converges to a point of the boundary component $E \in \mathcal{C}(X)$ if and only if $\left\{h\left(x_{i}\right)\right\}$ represents the end $\phi_{0}(E)$. Moreover, Proposition 4.5 provides a homeomorphism $\phi_{1}: \mathcal{E}(\Omega) \rightarrow \partial \Omega$ with the 
property that a sequence $\left\{y_{i}\right\} \in \mathcal{U}(X)$ represents the end $E \in \mathcal{E}(\Omega)$ if and only if it converges to the boundary point $\phi_{1}(E) \in \partial \Omega$. We define the extension of $h$ to $\partial X$ by setting $h(x)=\phi_{1} \circ \phi_{0}(E)$, where $E \in \mathcal{C}(X)$ is the boundary component containing $x \in \partial X$. The naturality properties of $\phi_{0}$ and $\phi_{1}$ ensure that $h: \bar{X} \rightarrow \mathbb{S}^{2}$ so defined is continuous. As $\left.h\right|_{X}$ is a homeomorphism onto $\Omega$ and $\partial \Omega=\mathbb{S}^{2} \backslash \Omega$, the definitions show that the extended map is a surjection.

Now, let $E \in \mathcal{C}(X)$ and $\epsilon>0$. By construction (or from the fact that $\left.h\right|_{X}$ is a homeomorphism and $\mathbb{S}^{2} \backslash \Omega$ is totally disconnected), the set $h(E)$ consists of a single point in $\partial \Omega$. Suppose that there is no $\epsilon^{\prime}>0$ such that (4.1) holds. Then there is a sequence of points

$$
x_{n} \in \bar{X} \backslash \mathcal{N}_{\bar{X}}(E, \epsilon)
$$

such that $\left\{h\left(x_{n}\right)\right\}$ converges to $h(E) \in \partial \Omega$. By compactness and the fact that $\left.h\right|_{X}$ is a homeomorphism onto $\Omega$, the sequence $\left\{x_{n}\right\}$ has a limit point $x \in \partial X \backslash \mathcal{N}_{\bar{X}}(E, \epsilon)$. This means that $x$ lies in some boundary component $F \neq E$. However, the continuity of $h$ implies that $h(F)=h(x)=h(E)$, which contradicts the fact that $\phi_{1} \circ \phi_{0}$ is injective.

Corollary 3.11 implies that $\mathcal{C}(X)$ is naturally homeomorphic to $\mathcal{E}(X)$, and Remark 3.3 shows that $\mathcal{E}(X)$ is naturally homeomoprhic to $\mathcal{E}(\Omega)$. Proposition 4.5 now yields the final statement of the theorem.

As an application of Proposition 4.1, we prove Proposition 2.7, which relates the ALLC condition and the relative separation of boundary components, and Proposition 2.8, which improves the LLC condition to the ALLC condition.

Proof of Proposition 2.7. Let $\Omega$ be a circle domain, and suppose that there is a number $c>0$ such that the components $\left\{E_{i}\right\}_{i \in I}$ of $\partial \Omega$ satisfy

$$
\triangle\left(E_{i}, E_{j}\right) \geq c
$$

whenever $i \neq j \in I$. Fix $\Lambda \geq 1$ so large that $2 c^{-1}<2 \Lambda^{2}-1$. We will show that $\Omega$ is $\Lambda$-ALLC. Let $p \in \Omega$ and $r>0$. It suffices to show that

$$
A_{\Omega}(p, r / \Lambda, 2 \Lambda r)
$$

is connected.

Let $h: \bar{\Omega} \rightarrow \mathbb{S}^{2}$ be the continuous surjection provided by Corollary 4.6. The complement of $h(\Omega)$ is a compact and totally disconnected set, and hence has topological dimension 0 (see Section II.4 in [17]). The definition of $\Lambda$ and (4.2) guarantee that there is at most one index $i \in I$ such that $E_{i}$ intersects both $\bar{B}_{\mathbb{S}^{2}}(p, r / \Lambda)$ and $\mathbb{S}^{2} \backslash B(p, 2 \Lambda r)$. This implies that $h\left(A_{\Omega}(p, r / \Lambda, 2 \Lambda r)\right)$ is the complement, in the domain bounded by two Jordan curves that touch at no more than one point, of a set of topological dimension 0. It is therefore connected (Theorem IV.4 in [17]). See Figure 2.

We leave the converse statement as an exercise for the reader, as it is not needed in this paper. 


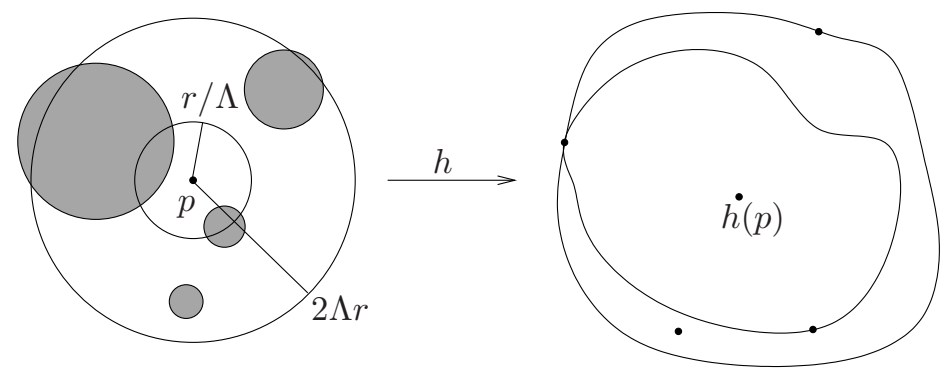

Figure 2. The ALLC property of circle domains.

In the proof of Proposition 2.8, we will use the following separation theorem of point-set topology (see Section V.9 in [17]).

Theorem 4.7 (Janiszewski). Suppose that $A$ and $B$ are closed subsets of $\mathbb{S}^{2}$ such that $\operatorname{card}(A \cap B) \leq 1$. If $y$ and $z$ are points of $\mathbb{S}^{2}$ that lie in the same component of $\mathbb{S}^{2} \backslash A$ and in the same component of $\mathbb{S}^{2} \backslash B$, then $y$ and $z$ lie in the same component of $\mathbb{S}^{2} \backslash(A \cup B)$.

Lemma 4.8. Let $(X, d)$ be a metric space homeomorphic to a domain in $\mathbb{S}^{2}$ that satisfies the $\mathrm{LLC}_{1}$-condition, and let $A$ and $B$ be disjoint closed subsets of $X$. Let $\mathcal{C}$ be the collection of components that intersect $\bar{A} \cap \bar{B}$, and assume that card $\mathcal{C} \leq 1$. If $u, v \in X$ are in the same component of $X \backslash A$ and in the same component of $X \backslash B$, then they are in the same component of $X \backslash(A \cup B)$.

Proof. Let $h: \bar{X} \rightarrow \mathbb{S}^{2}$ be the continuous surjection provided by Corollary 4.6. In particular $\left.h\right|_{X}$ is a homeomorphism onto a domain $\Omega=\mathbb{S}^{2} \backslash T$, where $T$ is a closed totally disconnected set. Moreover, $h$ maps each component of $\partial X$ to a distinct point of $T$. Hence, our assumptions imply that $h(\bar{A})$ and $h(\bar{B})$ are closed subsets of $\mathbb{S}^{2}$ such that $h(\bar{A}) \cap h(\bar{B})$ is either empty or a single point of $T$. Janiszewski's theorem now implies that $h(u)$ and $h(v)$ are in the same component $C$ of

$$
\mathbb{S}^{2} \backslash(h(\bar{A}) \cup h(\bar{B})) .
$$

Since $T \cap C$ is totally disconnected and a subset of the compact and totally disconnected set $T$, it has topological dimension 0 (see Section II.4 in [17]). This implies that $T \cap C$ does not disconnect $C$ (Theorem IV.4 in [17]), and hence $h(u)$ and $h(v)$ are contained in the same component of $\Omega \backslash(h(A) \cup h(B))$. Since $\left.h\right|_{X}$ is a homeomorphism onto $\Omega$, this yields the desired result.

Proof of Proposition 2.8. By Lemma 2.4, it suffices to consider a point $x \in X$ and radius $r>0$, and suppose that $y$ and $z$ are points of $A(x, r, 2 r)$. Denote by $\delta>0$ the minimum distance between components of $\partial X$, and set $s=\operatorname{diam} X / \delta$. By Proposition 2.3 we may assume that $X$ satisfies the $\lambda$-LLC condition for some $\lambda \geq 1$. 
We first assume that $r<\delta /(4 \lambda)$. Set

$$
A=S_{X}(x, 2 \lambda r) \text { and } B=\bar{B}_{X}(x, r /(2 \lambda)) .
$$

The $\lambda$-LLC-condition implies that $y$ and $z$ lie in the same component of $X \backslash A$ (namely, the component containing $x$ ) and in the same component of $X \backslash B$. The restriction on $r$ implies that there is at most one component $E$ of $\partial X$ that satisfies $E \cap(\bar{A} \cup \bar{B}) \neq \emptyset$. Hence, by Lemma 4.8, the points $y$ and $z$ are in the same component of $X \backslash(A \cup B)$. Since $(X, d)$ is locally path-connected, this implies that they are contained in a continuum in $A(x, r /(2 \lambda), 2 \lambda r)$.

Since $A(x, r, 2 r)$ is empty if $r>\operatorname{diam} X$, we may now assume that $\delta /(4 \lambda) \leq$ $r \leq \operatorname{diam} X$. The $\widetilde{\mathrm{LLC}_{1}}$ condition provides an embedding $\gamma:[0,1] \rightarrow X$ such that $\gamma(0)=y, \gamma(1)=z$, and $\operatorname{im} \gamma \subseteq B(x, 2 \lambda r)$. If there is no $t \in[0,1]$ such that $\gamma(t) \in B(x, r /(16 \lambda s))$, then the proof is complete. Otherwise, let

$$
\begin{aligned}
& t_{1}=\min \{t \in[0,1]: \gamma(t) \in S(x, r /(8 \lambda s))\}, \\
& t_{2}=\max \{t \in[0,1]: \gamma(t) \in S(x, r /(8 \lambda s))\} .
\end{aligned}
$$

Since $\gamma$ is an embedding, $X$ is connected, and $y, z \in A(x, r, 2 r)$, we see that $t_{1}<t_{2}$. As $r /(8 \lambda s)<\delta /(4 \lambda)$, we may apply the first case considered above to the points $\gamma\left(t_{1}\right)$ and $\gamma\left(t_{2}\right)$, producing a continuum $\Gamma \subseteq A\left(x, r /\left(16 \lambda^{2} s\right), r /(4 s)\right)$ that contains $\gamma\left(t_{1}\right)$ and $\gamma\left(t_{2}\right)$. Now, the continuum

$$
\gamma\left(\left[0, t_{1}\right]\right) \cup \Gamma \cup \gamma\left(\left[t_{2}, 1\right]\right) \subseteq A\left(x, r /\left(16 \lambda^{2} s\right), 2 \lambda r\right)
$$

contains $y$ and $z$.

\section{Crosscuts}

In this section, we assume that $X$ is a metric space that has compact completion, is homeomorphic to a domain in $\mathbb{S}^{2}$, and satisfies the $\lambda$-LLC $\mathrm{C}_{1}$ condition for some $\lambda \geq 1$.

A crosscut is an embedding $\gamma:[0,1] \rightarrow \bar{X}$ such that

$$
\gamma([0,1]) \cap \partial X=\gamma(0) \cup \gamma(1) .
$$

Note that if $\gamma$ is a crosscut, then $\left.\gamma\right|_{(0,1)}$ is a proper embedding. If $\gamma(0)$ and $\gamma(1)$ are distinct points that belong to the same component $E$ of $\partial X$, then we say that $\gamma$ is an E-crosscut.

The following proposition shows that under the assumption of the $\mathrm{LLC}_{1}$ condition, $E$-crosscuts behave as they do in the case of a circle domain in $\mathbb{S}^{2}$.

Proposition 5.1. Let $E$ be a component of $\partial X$, and let $\gamma$ be an E-crosscut. Then $X \backslash \operatorname{im} \gamma$ has precisely two components. Denoting these components by $U$ and $V$, there hold:

(i) for every $\epsilon>0$, there is a closed set $K \subseteq X$ with $\operatorname{dist}(E, K)>0$ such that $U \backslash K$ is a connected subset of $\mathcal{N}_{\bar{X}}(E, \epsilon) \cap U$, and the same statement is valid for $V$; 
(ii) the sets $\bar{U} \backslash \operatorname{im} \gamma$ and $\bar{V} \backslash \operatorname{im} \gamma$ are the components of $\bar{X} \backslash \operatorname{im} \gamma$, and $\bar{U} \cap \bar{V}=\operatorname{im} \gamma$;

(iii) the sets $\bar{U} \cap E$ and $\bar{V} \cap E$ are connected.

Proof. By Proposition 2.3, we may assume that $X$ in fact satisfies the $\lambda$-LLC condition.

Let $h: \bar{X} \rightarrow \mathbb{S}^{2}$ be the continuous surjection provided by Corollary 4.6 , and let $\Omega=h(X)$. Since $\left.h\right|_{X}$ is a homeomorphism, the map $h \circ \gamma:(0,1) \hookrightarrow \Omega$ is a proper embedding. As $h(E)$ is a single point and $h$ is continuous,

$$
\lim _{t \rightarrow 0} h \circ \gamma(t)=h(E)=\lim _{t \rightarrow 1} h \circ \gamma(t) .
$$

This implies that the continuous map $h \circ \gamma:[0,1] \rightarrow \Omega \cup\{h(E)\}$ defines a Jordan curve. The Jordan curve theorem states that $\mathbb{S}^{2} \backslash \operatorname{im}(h \circ \gamma)$ consists of two disjoint domains $\widetilde{U}$ and $\widetilde{V}$, each homeomorphic to $\mathbb{R}^{2}$, that have common boundary $\operatorname{im}(h \circ \gamma)$. Then $U:=h^{-1}(\widetilde{U} \cap \Omega)$ and $V:=h^{-1}(\widetilde{U} \cap \Omega)$ are disjoint nonempty open sets satisfying $U \cup V=X \backslash \operatorname{im} \gamma$. As $\left.h\right|_{X}$ is a homeomorphism, in order to show that $U$ and $V$ are the components of $X \backslash \operatorname{im} \gamma$, we need only show $\widetilde{U} \cap \Omega$ and $\widetilde{V} \cap \Omega$ are connected. Since $\mathbb{S}^{2} \backslash \Omega$ is compact and totally disconnected, it has topological dimension 0 (see Section II.4 in [17]). Thus $\widetilde{U} \cap \Omega$ is homeomorphic to the complement in $\mathbb{R}^{2}$ of a set of topological dimension 0 , and hence is connected (Theorem IV.4 in [17]). The same proof applies to $\widetilde{V} \cap \Omega$.

We proceed to the proof of (i). Let $\epsilon>0$. By Corollary 4.6, we may find $\epsilon^{\prime}>0$ such that

$$
h^{-1}\left(B_{\mathbb{S}^{2}}\left(h(E), \epsilon^{\prime}\right)\right) \subseteq \mathcal{N}_{\bar{X}}(E, \epsilon) .
$$

As $\operatorname{im}(h \circ \gamma)$ is a Jordan curve, by Schoenflies' theorem there is a homeomorphism $H: \mathbb{S}^{2} \rightarrow \mathbb{S}^{2}$ such that $H(\widetilde{U})$ is a standard ball in $\mathbb{S}^{2}$ whose boundary contains the point $H \circ h(E)$. By the continuity of $H^{-1}$ and (5.2) we may find $\epsilon^{\prime \prime}>0$ so small that

$$
h^{-1} \circ H^{-1}\left(B_{\mathbb{S}^{2}}\left(H \circ h(E), \epsilon^{\prime \prime}\right)\right) \subseteq \mathcal{N}_{\bar{X}}(E, \epsilon) .
$$

The set

$$
B_{\mathbb{S}^{2}}\left(H \circ h(E), \epsilon^{\prime \prime}\right) \cap H(\widetilde{U})
$$

is the nonempty intersection of two standard balls in $\mathbb{S}^{2}$ and hence is itself homeomorphic to $\mathbb{R}^{2}$. As before, Section II.4 and Theorem IV.4 in [17] imply that

$$
B_{\mathbb{S}^{2}}\left(H \circ h(E), \epsilon^{\prime \prime}\right) \cap H(\widetilde{U}) \cap H(\Omega)
$$

is connected. It now follows from $(5.3)$, the fact that $\left.h\right|_{X}$ is a homeomorphism onto $\Omega$, and the definition of $U$ that

$$
h^{-1} \circ H^{-1}\left(B_{\mathbb{S}^{2}}\left(H \circ h(E), \epsilon^{\prime \prime}\right)\right) \cap U
$$

is a connected subset of $\mathcal{N}_{\bar{X}}(E, \epsilon)$. We set

$$
K=X \backslash\left(h^{-1} \circ H^{-1}\left(B_{\mathbb{S}^{2}}\left(H \circ h(E), \epsilon^{\prime \prime}\right)\right)\right) .
$$

The continuity of $H \circ h$ now shows that $\operatorname{dist}(E, K)>0$. An analogous proof applies to $V$. 
We next address (ii). It follows from the definitions that $\bar{U} \backslash \operatorname{im} \gamma$ and $\bar{V} \backslash \operatorname{im} \gamma$ are nonempty, closed in $\bar{X} \backslash \operatorname{im} \gamma$, and satisfy

$$
(\bar{U} \backslash \operatorname{im} \gamma) \cup(\bar{V} \backslash \operatorname{im} \gamma)=\bar{X} \backslash \operatorname{im} \gamma .
$$

Moreover, as $\bar{U} \backslash \operatorname{im} \gamma$ is the topological closure in $\bar{X} \backslash \operatorname{im} \gamma$ of the connected set $U$, it is connected. Similarly, $\bar{V} \backslash \operatorname{im} \gamma$ is connected. Hence $\bar{U} \backslash \operatorname{im} \gamma$ and $\bar{V} \backslash \operatorname{im} \gamma$ are the components of $\bar{X} \backslash \operatorname{im} \gamma$.

As the common boundary of $\widetilde{U}$ and $\widetilde{V}$ is $\operatorname{im} h \circ \gamma$, we see that $\bar{U} \cap \bar{V} \supseteq \operatorname{im} \gamma$. Suppose there is a point $z \in \bar{X} \backslash \operatorname{im} \gamma$ that is an accumulation point of both $U$ and $V$. Let $0<\epsilon<\operatorname{dist}(z, \operatorname{im} \gamma) / \lambda$. By assumption we may find points $u \in$ $U \cap B_{\bar{X}}(z, \epsilon)$ and $v \in V \cap B_{\bar{X}}(z, \epsilon)$. The $\lambda$-LLC $\widetilde{L L}_{1}$ condition now implies that $u$ and $v$ can be connected in $X \backslash \operatorname{im} \gamma$, a contradiction. Hence $\bar{U} \cap \bar{V}=\operatorname{im} \gamma$.

To prove (iii), we show that $\bar{U} \cap E$ is connected; the corresponding statement for $V$ is proven in the same way. If $\bar{U} \cap E$ is not connected, then we may find disjoint, nonempty, and compact sets $A$ and $B$ such that $A \cup B=\bar{U} \cap E$. Fix $0<\epsilon<\operatorname{dist}(A, B) / 2$. We first claim that there is a number $\delta>0$ such that

$$
\mathcal{N}_{\bar{X}}(E, \delta) \cap U \subseteq \mathcal{N}_{\bar{X}}(A \cup B, \epsilon) \cap U .
$$

If this claim is false, then for every $n \in \mathbb{N}$ there are points $u_{n} \in U$ and $x_{n} \in E$ such that $d\left(x_{n}, u_{n}\right)<1 / n$ and $\operatorname{dist}\left(u_{n}, A \cup B\right) \geq \epsilon$. Since $E$ is compact, there is a subsequence of $\left\{x_{n}\right\}$ that converges to a point $x \in E$. It follows that the corresponding subsequence of $\left\{u_{n}\right\}$ converges to $x$ as well. Hence $x \in \bar{U} \cap E$ but $\operatorname{dist}(x, A \cup B) \geq \epsilon$, a contradiction. This proves the claim.

By (i), there is a closed set $K \subseteq X$ such that $\operatorname{dist}(E, K)>0$ and $U \backslash K$ is a connected subset of $\mathcal{N}_{\bar{X}}(E, \delta) \cap U$, and hence, by the claim, of $\mathcal{N}_{\bar{X}}(A \cup B, \epsilon) \cap U$. However, $\operatorname{since} \operatorname{dist}(E, K)>0$, we may find points

$$
a \in \mathcal{N}_{\bar{X}}(A, \epsilon) \cap(U \backslash K) \text { and } \quad b \in \mathcal{N}_{\bar{X}}(B, \epsilon) \cap(U \backslash K) .
$$

This is a contradiction since $\operatorname{dist}(A, B)>2 \epsilon$.

\section{Uniformization of the boundary components}

In this section, we assume that $X$ is a metric space that has compact completion, is homeomorphic to a domain in $\mathbb{S}^{2}$, and satisfies the full $\lambda$-LLC condition for some $\lambda \geq 1$.

We prove that each boundary component $E \in \mathcal{C}(X)$ with at least two points is homeomorphic to $\mathbb{S}^{1}$ and satisfies the $\lambda^{\prime}$-LLC condition, for some $\lambda^{\prime} \geq 1$ depending only on $\lambda$. To do so, we employ a recognition theorem of point-set topology: a metric space is homeomorphic to $\mathbb{S}^{1}$ if and only if it is a locally connected continuum such that removal of any one point results in a connected space, while the removal of any two points results in a space that is not connected [26]. This section adapts Section 4 of [27] to our setting; we omit certain proofs that need little or no translation. 
Proposition 6.1. Let $E$ be a component of $\partial X$, and let $p \in E$. Then $E \backslash\{p\}$ is connected.

Proof. As a single point set and the empty set are connected, we may assume that $E$ has at least three points. Let $x$ and $y$ be arbitrary distinct points of $E \backslash\{p\}$. It suffices to show that there is a connected subset of $E \backslash\{p\}$ that contains $x$ and $y$. By the $\widetilde{\mathrm{LLC}}_{1}$-condition, there is an E-crosscut $\gamma$ with $\gamma(0)=x$ and $\gamma(1)=y$. By Proposition 5.1 (ii), there is a unique component $U$ of $X \backslash \operatorname{im} \gamma$ such that $\bar{U} \cap E$ does not contain the point $p$. Then $x$ and $y$ are contained $\bar{U} \cap E$, which by Proposition 5.1 (iii) is a connected subset of $E \backslash\{p\}$.

Proposition 6.2. Let $E$ be a component of $\partial X$ with at least two points. If $p, q \in E$ are distinct points, then $E \backslash\{p, q\}$ is not connected.

Proof. See Prop. 4.11 in [27]. Here, Proposition 5.1 plays the role of Lemma 4.10 in $[27]$.

We now consider the local connectivity of boundary components of $X$. We will need a few technical lemmas.

Lemma 6.3. Let $E$ be a component of $\partial X$, and suppose that $\gamma$ and $\gamma^{\prime}$ are $E$ crosscuts with the property that there is a compact interval $I \subseteq(0,1)$ such that $\gamma(t)=\gamma^{\prime}(t)$ for all $t \notin I$. Then there is a closed subset $K \subseteq X$ with $\operatorname{dist}(E, K)>0$ such that if points $p$ and $q$ in $\bar{X} \backslash K$ are in a single component of $\bar{X} \backslash \operatorname{im} \gamma$, then they are in a single component of $\bar{X} \backslash \operatorname{im} \gamma^{\prime}$.

Proof. See Lemma 4.12 in [27].

We will briefly need a notion of transversality. Let $Y$ be a topological space homeomorphic to a domain in $\mathbb{S}^{2}$, and let $\alpha, \beta:(0,1) \rightarrow Y$ be embeddings. Given $x \in \operatorname{im} \alpha \cap \operatorname{im} \beta$, we say that $\alpha$ and $\beta$ intersect transversally at $x$ if there is an open neighborhood $U$ of $x$ and a homeomorphism $h: U \rightarrow \mathbb{R}^{2}$ such that $h(U \cap \operatorname{im} \alpha)$ is the $x$-axis and $h(U \cap \operatorname{im} \beta)$ is the $y$-axis.

Lemma 6.4. Let $a, b, p$, and $q$ be distinct points on a Jordan curve $\alpha \subseteq \mathbb{S}^{2}$, and let $U \subseteq \mathbb{S}^{2}$ be a simply connected domain with boundary $\alpha$. If $V$ is any open subset of $\bar{U}$ containing $\alpha$, then there are embeddings $\alpha_{a b}:[0,1] \rightarrow V$ and $\alpha_{p q}:[0,1] \rightarrow V$ connecting $a$ to $b$ and $p$ to $q$ respectively, such that either $\operatorname{im} \alpha_{a b} \cap \operatorname{im} \alpha_{p q}=\emptyset$, or $\alpha_{a b}$ and $\alpha_{p q}$ have a single intersection, and that intersection is in $U$ and is transverse.

Proof. By the Schönflies theorem, there is a homeomorphism $H: \mathbb{S}^{2} \rightarrow \mathbb{S}^{2}$ such that $H(\alpha)$ is a round circle. Then $H(V)$ is open in $H(\bar{U})$ and it contains a round annulus that has $H(\alpha)$ as a boundary component. Clearly $H(a), H(b), H(p)$, and $H(q)$ may be connected as desired inside this annulus. Taking inverse images under $H$ now yields the desired result. 
Lemma 6.5. Let $a, b, p$, and $q$ be distinct points of a component $E$ of $\partial X$, and let $\gamma_{a b}$ and $\gamma_{p q}$ be E-crosscuts connecting a to $b$ and $p$ to $q$, respectively. If $p$ and $q$ are contained in a single component of $\bar{X} \backslash \gamma_{a b}$, then $a$ and $b$ are contained in $a$ single component of $\bar{X} \backslash \gamma_{p q}$.

Proof. The fact that the points $a, b, p$, and $q$ are all distinct implies that $K=$ $\operatorname{im} \gamma_{a b} \cap \operatorname{im} \gamma_{p q}$ is a compact subset of $X$. If $K$ is empty, then $\gamma_{a b}$ connects $a$ to $b$ without intersecting $\gamma_{p q}$, as desired. Hence we may assume that $K \neq \emptyset$.

By Corollary 4.6, there is a continuous surjection $h: \bar{X} \rightarrow \mathbb{S}^{2}$ where $\left.h\right|_{X}$ is a homeomorphism onto a domain $\Omega$ in $\mathbb{S}^{2}$ with totally disconnected complement.

Let $\Omega_{1} \subseteq \Omega_{2} \subseteq \cdots$ be the exhaustion of $\Omega$ guaranteed to exist by Proposition 3.7. Since $a, b, p$, and $q$ are all elements of the same boundary component $E$, for each $i \in \mathbb{N}$ there is a single simply connected component $U_{i}$ of $\mathbb{S}^{2} \backslash \Omega_{i}$ containing $h(\{a, b, p, q\})$. Fix $i \in \mathbb{N}$ so large that $h(K) \subseteq \Omega_{i}$. Then we may find parameters $t_{1}, t_{2}, s_{1}, s_{2} \in(0,1)$ such that

$$
\begin{aligned}
& t_{1}=\min \left\{t \in[0,1]: h \circ \gamma_{a b}(t) \in \partial U_{i}\right\}, \\
& t_{2}=\max \left\{t \in[0,1]: h \circ \gamma_{a b}(t) \in \partial U_{i}\right\}, \\
& s_{1}=\min \left\{s \in[0,1]: h \circ \gamma_{p q}(t) \in \partial U_{i}\right\}, \\
& s_{2}=\max \left\{s \in[0,1]: h \circ \gamma_{p q}(t) \in \partial U_{i}\right\} .
\end{aligned}
$$

Since $\Omega_{i}$ has only finitely many boundary components and $h(K)$ is a compact subset of $\Omega_{i}$, we may find a relatively open neighborhood $V$ of $\partial U_{i}$ in $\mathbb{S}^{2} \backslash U_{i}$ such that $V \subseteq \Omega_{i} \backslash h(K)$. By Lemma 6.4, there are embeddings $\alpha_{a b}:[0,1] \rightarrow V$ and $\alpha_{p q}:[0,1] \rightarrow V$ connecting $h \circ \gamma_{a b}\left(t_{1}\right)$ to $h \circ \gamma_{a b}\left(t_{2}\right)$ and $h \circ \gamma_{p q}\left(s_{1}\right)$ to $h \circ \gamma_{p q}\left(s_{2}\right)$ respectively, such that either $\operatorname{im} \alpha_{a b} \cap \operatorname{im} \alpha_{p q}=\emptyset$, or $\alpha_{a b}$ and $\alpha_{p q}$ have a single transversal intersection.

Let $\widetilde{\gamma}_{a b}$ be the path defined by concatenating $\left.\gamma_{a b}\right|_{\left[0, t_{1}\right]}, h^{-1} \circ \alpha_{a b}$, and $\left.\gamma_{a b}\right|_{\left[t_{2}, 1\right]}$. Similarly define $\widetilde{\gamma}_{p q}$. Then either im $\widetilde{\gamma}_{a b}$ and im $\widetilde{\gamma}_{p q}$ are disjoint, or they have a single intersection, and that intersection is transversal and located in $X$. In the former case, $a$ and $b$ are in a single component of $\bar{X} \backslash \widetilde{\gamma}_{p q}$, and so Lemma 6.3 provides the desired result. In the latter case, the transversality and Proposition 5.1 (ii) imply that $p$ and $q$ are not contained in a connected subset of $\bar{X} \backslash \widetilde{\gamma}_{a b}$. Lemma 6.3 shows that this is a contradiction.

Proposition 6.6. Let $E$ be a component of $\partial X$. Then $E$ satisfies the $4 \lambda^{4}-\mathrm{LLC}_{1}$ condition. In particular, $E$ is locally connected.

Proof. We may assume that $E$ has at least two points. Let $p \in E$, and $r>0$. It suffices to find a continuum $F$ such that

$$
B_{\bar{X}}(p, r) \cap E \subseteq F \subseteq B_{\bar{X}}\left(p, 4 \lambda^{4} r\right) \cap E .
$$

As $E$ itself is connected, we may assume that there is some point

$$
q \in E \backslash B_{\bar{X}}\left(p, 4 \lambda^{4} r\right) .
$$


The $\widetilde{\mathrm{LLC}}_{1}$ condition provides an E-crosscut $\gamma_{p q}$ connecting $p$ to $q$. Let $U$ and $V$ be the components of $X \backslash \operatorname{im} \gamma_{p q}$, and set $A=\bar{U} \cap E$ and $B=\bar{V} \cap E$. By Proposition 5.1 (iii), $A$ and $B$ are connected. As $\{p, q\}=A \cap B$ and $d(p, q)>4 \lambda^{4} r$, we may find distinct points $a \in A$ and $b \in B$ such that $d(p, a)=2 \lambda^{2} r$ and $d(p, b)=2 \lambda^{2} r$. The $\lambda-\widetilde{\mathrm{LLC}}_{1}$ condition provides a crosscut $\gamma_{a b}$ connecting $a$ to $b$ with im $\gamma_{a b} \subseteq B_{\bar{X}}\left(p, 3 \lambda^{3} r\right)$. By Proposition 5.1 (ii), there is a unique component $W$ of $X \backslash \operatorname{im} \gamma_{a b}$ with $p \in \bar{W} \cap E$. Set $F:=\bar{W} \cap E$. Applying Proposition 5.1 (iii) again, we see that the set $F$ is connected.

We first show that $F \subseteq B_{\bar{X}}\left(p, 4 \lambda^{4} r\right) \cap E$. Suppose that there is a point $x \in$ $F \backslash B_{\bar{X}}\left(p, 4 \lambda^{4} r\right)$. By the $\lambda$-LLC 2 condition, there is a path connecting $x$ to $q$ without intersecting $B_{\bar{X}}\left(p, 4 \lambda^{3} r\right)$. This implies that $p$ and $q$ are in the same component of $\bar{X} \backslash \gamma_{a b}$. However, by Proposition 5.1 (ii), the points $a$ and $b$ lie in different components of $\bar{X} \backslash \operatorname{im} \gamma_{p q}$. This contradicts Lemma 6.5.

We now show that $B_{\bar{X}}(p, r) \cap E \subseteq F$. Since $\gamma_{a b}$ is continuous, we may find parameters $0<t_{a}<1$ and $0<t_{b}<1$ such that

$$
\operatorname{diam}\left(\gamma_{a b}\left(\left[0, t_{a}\right]\right)\right) \leq \lambda^{2} r \quad \text { and } \quad \operatorname{diam}\left(\gamma_{a b}\left(\left[t_{b}, 1\right]\right)\right) \leq \lambda^{2} r .
$$

Set $a^{\prime}=\gamma_{a b}\left(t_{a}\right)$ and $b^{\prime}=\gamma_{a b}\left(t_{b}\right)$. Then $a^{\prime}, b^{\prime} \in X \backslash B_{\bar{X}}\left(p, \lambda^{2} r\right)$, and so the $\lambda$-LLC $\widetilde{\mathrm{LC}}_{2}$ condition provides an embedding $\gamma_{a^{\prime} b^{\prime}}:[0,1] \rightarrow X$ such that $\gamma_{a^{\prime} b^{\prime}}(0)=a^{\prime}, \gamma_{a^{\prime} b^{\prime}}(1)$ $=b^{\prime}$, and $\operatorname{im} \gamma_{a^{\prime} b^{\prime}} \subseteq X \backslash B \bar{X}(p, \lambda r)$. The set

$$
S=\gamma_{a b}\left(\left[0, t_{a}\right]\right) \cup \operatorname{im} \gamma_{a^{\prime} b^{\prime}} \cup \gamma_{a b}\left(\left[t_{b}, 1\right]\right)
$$

does not intersect $B_{\bar{X}}(p, \lambda r)$, and is the image of a path in $\bar{X}$. Since the image of a path in $\bar{X}$ is arc-connected, we may find a crosscut $\gamma^{\prime}$ connecting $a$ to $b$ with $\operatorname{im} \gamma^{\prime} \subseteq S$. Furthermore, we may find a compact interval $I \subseteq(0,1)$ such that $\gamma^{\prime}(t)=\gamma_{a b}(t)$ for all $t \in[0,1] \backslash I$. Suppose that there is a point $x \in B_{\bar{X}}(p, r) \cap E$ that is not contained in $F$. Then $x$ and $p$ are in different components of $\bar{X} \backslash \operatorname{im} \gamma_{a b}$. By Lemma 6.3, this implies that $x$ and $p$ are in different components $\bar{X} \backslash \operatorname{im} \gamma^{\prime}$. However, the $\lambda$-LLC $\widetilde{\mathrm{LC}}_{1}$ condition shows that $x$ and $p$ may be connected by an arc contained in $B_{\bar{X}}(p, \lambda r)$. This is a contradiction.

Proof of Theorem 1.6. We suppose that $X$ is a metric space homeomorphic to a domain in $\mathbb{S}^{2}$, has compact completion, and satisfies the $\lambda$-LLC condition, $\lambda \geq 1$. By Proposition 2.3, $X$ in fact satisfies the $\lambda^{\prime}$-LLC condition for some $\lambda^{\prime}$ depending only on $\lambda$. Let $E$ be a component of $\partial X$ with at least two points. As $\bar{X}$ is compact, $E$ is a continuum. Hence, Propositions $6.1,6.2$, and 6.6 along with the recognition theorem of [26] show that $E$ is a topological circle. Proposition 6.6 shows that $E$ is $4 \lambda^{\prime 4}-\mathrm{LLC}_{1}$. The desired statement now follows from Proposition 4.15 in [27] and the characterization of quasicircles given in [25].

Remark 6.7. Let $E$ be a component of $\partial X$, let $\gamma$ be an $E$-crosscut, and let $U$ and $V$ be the components of $X \backslash \operatorname{im} \gamma$. By Proposition 5.1 (ii) and (iii), the sets $\bar{U} \cap E$ and $\bar{V} \cap E$ are connected and have intersection $\{\gamma(0), \gamma(1)\}$. Since Theorem 1.6 implies that $E$ is a topological circle, we may conclude that $(\bar{U} \cap E) \backslash \operatorname{im} \gamma$ and $(\bar{U} \cap E) \backslash \operatorname{im} \gamma$ are the components of $E \backslash \operatorname{im} \gamma$. 


\section{Topological uniformization of the completion}

In this section, in which the notation and assumptions are as in the previous section, we give the following topological uniformization for the completion of $X$, at least in the case of finitely many boundary components.

Theorem 7.1. Let $N \in \mathbb{N}$, and assume that $\partial X$ has $N$ components, all of which are nontrivial. Then the completion $\bar{X}$ is homeomorphic to the closure of a circle domain that has $N$ boundary components, all of which are nontrivial.

Remark 7.2. The homeomorphism type of a metric space $X$ and the homeomorphism type of the boundary $\partial X$ do not, in general, determine the homeomorphism type of the completion $\bar{X}$. The following example demonstrates this. The real projective plane $\mathbb{R} P^{2}$, formed by identifying antipodal points of $\mathbb{S}^{2}$, can be metrized as a subset of $\mathbb{R}^{4}$ endowed with the standard metric. Let $X \subseteq \mathbb{R} P^{2} \subseteq \mathbb{R}^{4}$ be the image of the sphere minus the equator under the identification of antipodal points. Then $X$ is homeomorphic to the disk, and $\partial X$ is homeomorphic to the image of the equator under the identification of antipodal points, i.e., it is homeomorphic to the circle. However, the completion $\bar{X}$ is homeomorphic to all of $\mathbb{R} P^{2}$, and not the closed disk. The LLC condition prevents this phenomena from occurring in the setting we are most interested in.

The proof of Theorem 7.1 relies on the following topological characterization of the closed disk, due to Zippin [29]. Here, given a topological space $Y$, an embedding $\alpha:[0,1] \rightarrow Y$ is said to span a subset $J$ of $Y$ if $\operatorname{im} \alpha \cap J=\{\alpha(0), \alpha(1)\}$ and $\alpha(0) \neq \alpha(1)$. Note that an embedding spans a component $E$ of the boundary $\partial X$ if and only if it is an $E$-crosscut.

Theorem 7.3 (Zippin). A locally connected and metrizable continuum $Y$ is homeomorphic to the closed disk $\overline{\mathbb{D}^{2}}$ if and only if $Y$ contains a topological circle $J$ with the following properties:

- there is an embedding $\alpha:[0,1] \rightarrow Y$ that spans $J$,

- for every embedding $\alpha:[0,1] \rightarrow Y$ spanning $J$, the set $Y \backslash \operatorname{im} \alpha$ is not connected,

- for every embedding $\alpha:[0,1] \rightarrow Y$ spanning $J$ and every closed subset $I \subsetneq$ $[0,1]$, the set $Y \backslash \alpha(I)$ is connected.

Remark 7.4. The homeomorphism constructed in the proof of Theorem 7.3 maps the Jordan curve $J$ onto the unit circle.

The key point of the proof of Theorem 7.1 is given in the following statement, which the reader may wish to compare to Remark 7.2 .

Lemma 7.5. Let $E \in \mathcal{C}(X)$ be an isolated and nontrivial component of $\partial X$, and let $h: \bar{X} \rightarrow \mathbb{S}^{2}$ be the continuous surjection provided by Corollary 4.6. Then for all sufficiently small $\epsilon>0$, the set $U=h^{-1}\left(\bar{B}_{\mathbb{S}^{2}}(h(E), \epsilon)\right)$ is homeomorphic to a closed annulus. 
Proof. Corollary 4.6 states that $h$ induces a homeomorphism from $\mathcal{C}(X)$ to $\partial(h(X))$. Since $E$ is isolated, it follows that $U \cap \partial X=E$ when $\epsilon$ is sufficiently small.

Fix such an $\epsilon$, and set $p=h(E) \in \mathbb{S}^{2}$ and $\alpha_{0}=h^{-1}\left(S_{\mathbb{S}^{2}}(p, \epsilon)\right)$. Then $\alpha_{0}$ is a Jordan curve in $X$. Equip

$$
U \coprod\left(\mathbb{S}^{2} \backslash B_{\mathbb{S}^{2}}(p, \epsilon)\right)
$$

with the disjoint union topology, and consider the quotient topological space

$$
Y=\left(U \coprod\left(\mathbb{S}^{2} \backslash B_{\mathbb{S}^{2}}(p, \epsilon)\right)\right) / x \sim h(x), \quad x \in \alpha_{0} .
$$

The result will follow once it is shown that $Y$ is homeomorphic to the closed disk, with $\pi(E)$ corresponding to the unit circle. To do so, we employ Theorem 7.3.

Let $\pi$ denote the usual projection map onto $Y$, and note that

$$
\left.\pi\right|_{U} \text { and }\left.\pi\right|_{\mathbb{S}^{2} \backslash B_{\mathbb{S}^{2}}(p, \epsilon)}
$$

are embeddings, and $\left.\pi\right|_{\alpha_{0}}$ is a two-to-one map. After some effort, it can be seen that $Y$ is compact, second-countable, and regular. The $\widetilde{\text { LLC }}$-condition on $\bar{X}$ also implies that $Y$ is connected and locally path-connected. By Urysohn's metrization theorem, it is also metrizable. Moreover, as $\left.h\right|_{X}$ is a homeomorphism, the set $U \backslash E$ is homeomorphic to $\bar{B}_{\mathbb{S}^{2}}(p, \epsilon) \backslash\{p\}$. Thus, elementary point-set topology shows that $Y \backslash \pi(E)$ is homeomorphic to $\mathbb{S}^{2} \backslash\{p\}$, i.e., to the plane.

By Theorem 1.6, the set $\pi(E)$ is a topological circle in $Y$. The existence of an embedding $\alpha:[0,1] \rightarrow Y$ that spans $\pi(E)$ follows from the $\widetilde{\text { LLC }}$ condition on $X$. We next check that any embedding $\alpha:[0,1] \rightarrow Y$ that spans $\pi(E)$ disconnects $Y$. Towards a contradiction, suppose that $Y \backslash \alpha$ is connected. Since $Y$ is locally pathconnected and Hausdorff, it follows that $Y \backslash \alpha$ is arc-connected.

We assume that the image of $\alpha$ intersects the circle $\pi\left(\alpha_{0}\right)$; the argument in the case that this does not occur is a simpler version of what follows. Set

$$
t_{0}=\min \left\{t \in[0,1]: \alpha(t) \in \pi\left(\alpha_{0}\right)\right\} \quad \text { and } \quad t_{1}=\max \left\{t \in[0,1]: \alpha(t) \in \pi\left(\alpha_{0}\right)\right\} .
$$

Then $0<t_{0} \leq t_{1}<1$. Denote

$$
\begin{aligned}
& p=\alpha(0) \in \pi(E), \\
& q=\alpha(1) \in \pi(E), \\
& p^{\prime}=\alpha\left(t_{0}\right) \in \pi\left(\alpha_{0}\right), \\
& q^{\prime}=\alpha\left(t_{1}\right) \in \pi\left(\alpha_{0}\right),
\end{aligned}
$$

and let $A$ be an arc of the topological circle $\pi\left(\alpha_{0}\right)$ with endpoints $p^{\prime}$ and $q^{\prime}$. It is possible that $A$ is a single point. Let $\alpha^{\prime}:[0,1] \rightarrow Y$ be an embedding such that $\alpha^{\prime}(t)=\alpha(t)$ when $t \in\left[0, t_{0}\right] \cup\left[t_{1}, 1\right]$, and such that $\alpha^{\prime}:\left[t_{0}, t_{1}\right] \rightarrow Y$ is an embedding parametrizing $A$. Then $\operatorname{im}\left(\alpha^{\prime}\right) \subseteq \pi(U)$, and hence $\alpha^{\prime}$ defines an $E$-crosscut $\widetilde{\alpha}$ in $\bar{X}$. By Remark 6.7, we may find points $x$ and $y$ in $E$ that are in different components of $\bar{X} \backslash \widetilde{\alpha}$. Since

$$
\operatorname{im} \alpha \cap \pi(E)=\pi(\operatorname{im} \widetilde{\alpha} \cap E),
$$

the points $\pi(x)$ and $\pi(x)$ are in $Y \backslash \alpha$. 
Our assumption now provides an embedding $\gamma:[0,1] \rightarrow Y \backslash \alpha$ such that $\gamma(0)=$ $\pi(x)$ and $\gamma(1)=\pi(y)$. Since $Y \backslash \pi(E)$ is homeomorphic to the plane $\mathbb{R}^{2}$, the Schoenflies theorem implies there is a homeomorphism $\Phi: Y \backslash \pi(E) \rightarrow \mathbb{R}^{2}$ that sends $\left.\alpha\right|_{(0,1)}$ to the real line. Since $\pi(x)$ and $\pi(y)$ are in $\pi(E)$, the embedding $\left.\Phi \circ \gamma\right|_{(0,1)}$ is proper. Moreover, its image does not intersect the real line. Let $B$ be a ball in $\mathbb{R}^{2}$ that contains the compact set $\Phi\left(\pi\left(\alpha_{0}\right)\right)$. As $\left.\Phi \circ \gamma\right|_{(0,1)}$ is proper, we may find points $s_{0}, s_{1} \in(0,1)$ such that if $t \notin\left(s_{0}, s_{1}\right)$, then $\Phi \circ \gamma(t) \notin B$. From the geometry of $\mathbb{R}^{2}$, we see that there is a path

$$
\beta:\left[s_{0}, s_{1}\right] \rightarrow \mathbb{R}^{2} \backslash(B \cup \mathbb{R} \times\{0\}) .
$$

Then $\operatorname{im} \beta$ does not intersect $\operatorname{im}\left(\Phi \circ \alpha^{\prime}\right)$. Let $\gamma^{\prime}$ denote the concatenation of $\left.\gamma\right|_{\left[0, s_{0}\right]}$, $\Phi^{-1} \circ \beta$, and $\left.\gamma\right|_{\left[s_{1}, 1\right]}$. Then im $\gamma^{\prime}$ contains $\pi(x)$ and $\pi(y)$ and is contained in $\pi(U) \backslash \alpha^{\prime}$. It follows that $\operatorname{im}\left(\pi^{-1}\left(\gamma^{\prime}\right)\right)$ connects $x$ to $y$ inside of $\bar{X} \backslash \widetilde{\alpha}$, contradicting the definition of $x$ and $y$. See Figure 3 .

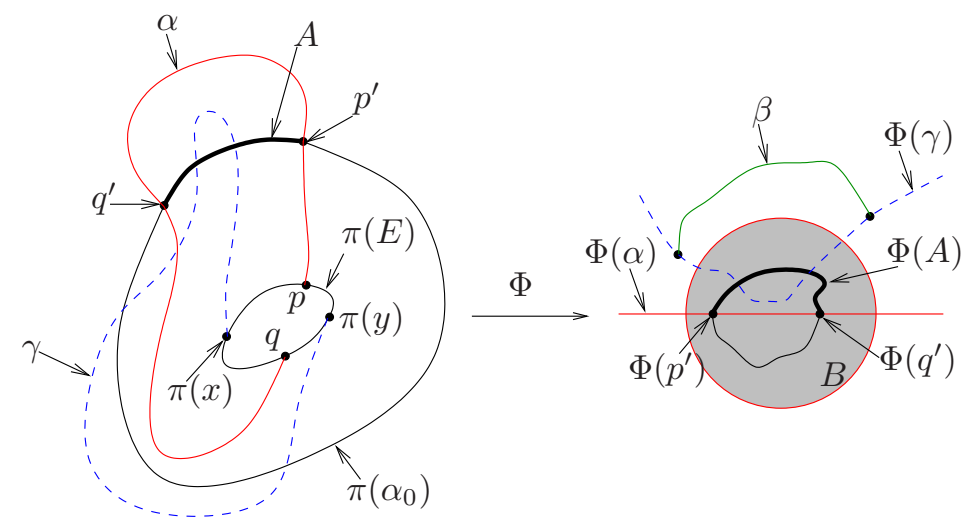

Figure 3. The proof of Lemma 7.5.

Finally, we check that if $I \subsetneq[0,1]$ is closed, then $\alpha(I)$ does not separate the $Y$. Since $Y$ is locally path-connected, it suffices to show that any pair of points $x, y \in$ $Y \backslash \pi(E)$ can be connected without intersecting $\alpha(I)$. This follows easily from the Schönflies theorem.

We will also need the following well-known topological statement, the proof of which is left to the reader.

Lemma 7.6. Let $\epsilon>0$ and $p \in \mathbb{S}^{2}$. Given any homeomorphism $\phi: S_{\mathbb{S}^{2}}(p, \epsilon) \rightarrow$ $S_{\mathbb{S}^{2}}(p, \epsilon)$, there is a homeomorphism $\Phi: A_{\mathbb{S}^{2}}(p, \epsilon, 2 \epsilon) \rightarrow A_{\mathbb{S}^{2}}(p, \epsilon, 2 \epsilon)$ such that $\left.\Phi\right|_{S_{\mathbb{S}^{2}}(p, \epsilon)}=\phi$ and $\left.\Phi\right|_{S_{\mathbb{S}^{2}}(p, 2 \epsilon)}$ is the identity mapping.

Given a metric space $Y$ homeomorphic to a domain in $\mathbb{S}^{2}$, we denote by $\mathcal{N}(Y) \subseteq$ $\mathcal{C}(Y)$ the collection of nontrivial components of $\partial Y$, and by $\mathcal{I}(Y) \subseteq \mathcal{N}(Y)$ the collection of nontrivial components of $\partial Y$ that are isolated as points in $\mathcal{C}(Y)$.

Theorem 7.1 is a special case of the following result. 
Theorem 7.7. Set

$$
\widetilde{X}=X \cup\left(\bigcup_{E \in \mathcal{I}(X)} E\right) .
$$

Then there are a circle domain $\Omega^{\prime} \subseteq \mathbb{S}^{2}$ and a homeomorphism

$$
\widetilde{h}: \tilde{X} \rightarrow \Omega^{\prime} \cup\left(\bigcup_{F \in \mathcal{N}\left(\Omega^{\prime}\right)} F\right) .
$$

Moreover, $\Omega^{\prime}$ and $\widetilde{h}$ may be chosen so that $\widetilde{h}$ induces a homeomorphism from $\mathcal{C}(X)$ to $\mathcal{C}\left(\Omega^{\prime}\right)$.

Proof. Let $h: \bar{X} \rightarrow \mathbb{S}^{2}$ denote the continuous surjection provided by Corollary 4.6. Let $E \in \mathcal{I}(X)$. By Lemma 7.5, there are a number $\epsilon_{E}>0$ and a homeomorphism

$$
h_{E}: h^{-1}\left(\bar{B}_{\mathbb{S}^{2}}\left(h(E), \epsilon_{E} / 2\right)\right) \rightarrow \bar{A}_{\mathbb{S}^{2}}\left(h(E), \epsilon_{E} / 4, \epsilon_{E} / 2\right) .
$$

Denote the domain of $h_{E}$ by $U_{E}$, and set $V_{E}=h^{-1}\left(\bar{B}_{\mathbb{S}^{2}}\left(h(E), \epsilon_{E}\right)\right)$. We may assume that the Jordan curve $\beta_{E}:=h^{-1}\left(S_{\mathbb{S}^{2}}\left(h(E), \epsilon_{E}\right)\right)$ is mapped onto $S_{\mathbb{S}^{2}}\left(h(E), \epsilon_{E}\right)$. Moreover, we may choose the number $\epsilon_{E}$ so small that $V_{E} \cap \partial X=E$, and that the resulting collection $\left\{V_{E}\right\}_{E \in \mathcal{I}(X)}$ is pairwise disjoint.

According to Lemma 7.6, for each $E \in \mathcal{I}(X)$, there is a homeomorphism $\Phi_{E}$ of $A_{\mathbb{S}^{2}}\left(h(E), \epsilon_{E} / 2, \epsilon_{E}\right)$ to itself that agrees with $h_{E} \circ h^{-1}$ on $S_{\mathbb{S}^{2}}\left(h(E), \epsilon_{E} / 2\right)$ and is the identity on $S_{\mathbb{S}^{2}}\left(h(E), \epsilon_{E}\right)$.

Define

$$
\Omega^{\prime}=h(X) \backslash\left(\bigcup_{E \in \mathcal{I}(X)} \bar{B}_{\mathbb{S}^{2}}\left(h(E), \epsilon_{E} / 4\right)\right) .
$$

Then the collection of nontrivial boundary components of $\Omega^{\prime}$ is given by $\mathcal{N}\left(\Omega^{\prime}\right)=$ $\left\{S_{\mathbb{S}^{2}}\left(h(E), \epsilon_{E} / 4\right)\right\}_{E \in \mathcal{I}(X)}$. The map $\widetilde{h}: \widetilde{X} \rightarrow \Omega^{\prime} \cup\left(\bigcup_{F \in \mathcal{N}\left(\Omega^{\prime}\right)} F\right)$ defined by

$$
\widetilde{h}(x)= \begin{cases}h(x) & x \notin \bigcup_{E \in \mathcal{I}(X)} V_{E}, \\ \Phi_{E} \circ h(x) & x \in V_{E} \backslash U_{E}, \\ h_{E}(x) & x \in U_{E}\end{cases}
$$

now yields the desired homeomorphism. See Figure 4.

The final assertion follows from the construction and the fact that there is a natural homeomorphism from $\mathcal{C}(h(X))$ to $\mathcal{C}\left(\Omega^{\prime}\right)$.

\section{ALLC and porous quasicircles}

A subset $Z$ of a metric space $(X, d)$ is $C$-porous, $C \geq 1$, if for every $z \in Z$ and $0<r \leq \operatorname{diam} X$, there is a point $x \in X$ such that

$$
B\left(x, \frac{r}{C}\right) \subseteq B(z, r) \backslash Z .
$$

Porous subsets are small compared to the ambient space in a quantitative sense. See Section 5.8 in [9] and Lemma 3.12 in [3] for a proof of the following well-known theorem. 


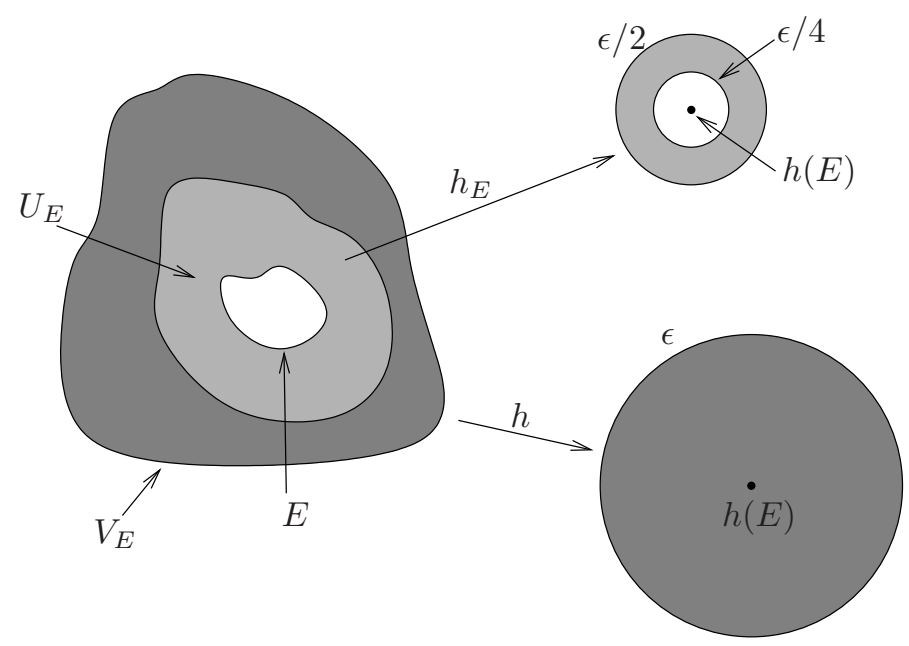

FIgURE 4. Topological uniformization of the completion.

Theorem 8.1. Let $(X, d)$ be an Ahlfors $Q$-regular metric space, $Q>0$, and let $\Gamma$ be a subset of $X$. Then $\Gamma$ is $C$-porous for some $C \geq 1$ if and only $\Gamma$ is $\left(\alpha, C^{\prime}\right)$ homogeneous for some $\alpha<Q$ and $C^{\prime} \geq 1$, quantitatively.

We recall that a quasicircle is any metric space that is quasisymmetrically equivalent to $\mathbb{S}^{1}$.

Theorem 8.2. Let $(X, d)$ be an ALLC metric space, and let $\Gamma \subseteq X$ be a quasicircle. Then $\Gamma$ is porous in $X$, quantitatively.

Proof. We suppose that $(X, d)$ is $\Lambda$-ALLC, $\Lambda \geq 1$, and that $\Gamma \subseteq X$ is a Jordan curve satisfying the following condition: there is $\lambda \geq 1$ such that for each pair of distinct points $x$ and $y$ on $\Gamma$

$$
\operatorname{diam}(I) \leq \lambda d(x, y)
$$

where $I$ is a component of $\Gamma \backslash\{x, y\}$ of minimal diameter. When $\Gamma$ is doubling, this condition is quantitatively equivalent to the assumption that $\Gamma$ is a quasicircle; see $[25]$.

Let $z \in \Gamma$ and $0<r \leq \operatorname{diam} X$. We consider three cases.

Case 1: $0<r<(\operatorname{diam} \Gamma) /(4 \Lambda)$. We may find a point $w \in \Gamma$ such that $d(z, w) \geq$ $2 \Lambda r$. We may also find points $u, v \in \Gamma$ such that $\{z, v, w, u\}$ is cyclically ordered on $\Gamma, d(z, u)=r /(4 \Lambda)=d(z, v)$, and if $J(z)$ is the component of $\Gamma \backslash\{u, v\}$ that contains $z$, then $J(z) \subseteq B(z, r /(4 \Lambda))$.

The $\Lambda$-ALLC condition on the space $X$ implies the existence of a continuum $\alpha \subseteq A\left(z, r /\left(8 \Lambda^{2}\right), r / 2\right)$ that contains $u$ and $v$. Let $J(u)$ be the component of $\Gamma \backslash\{z, w\}$ containing $u$, and let $I(u)=J(u) \cup\{z, w\}$. Define $J(v)$ and $I(v)$ similarly. 
We claim that $\operatorname{dist}(v, I(u)) \geq r /(8 \lambda \Lambda)$. If not, then the $\lambda$-three point condition implies that either $z$ or $w$ is within a distance of $r /(8 \Lambda)$ of $v$, which is not the case. Now, the connectedness of $\alpha$ implies that there is a point $x \in \alpha$ such that

$$
\operatorname{dist}(x, I(u))=\frac{r}{s}, \quad \text { where } s=8(2 \lambda+1) \Lambda^{2} .
$$

Suppose that there is a point $y \in I(v)$ such that $d(x, y)<r / s$. Then $\operatorname{dist}(y, I(u))<$ $2 r / s$, and hence the $\lambda$-three point condition implies that either $z$ or $w$ is within a distance $2 \lambda r / s$ of $y$, and hence

$$
\operatorname{dist}(x,\{w, z\})<\frac{2 \lambda r}{s}+\frac{r}{s}=\frac{r}{8 \Lambda^{2}} .
$$

Combined with the facts that $x \in \alpha \subseteq A\left(z, r /\left(8 \Lambda^{2}\right), r / 2\right)$ and $d(z, w) \geq 2 \Lambda r$, this yields a contradiction. Hence $\operatorname{dist}(x, \Gamma) \geq r / s$, and so the fact that $d(x, z)<r / 2$ implies

$$
B\left(x, \frac{r}{s}\right) \subseteq B(z, r) \backslash \Gamma
$$

Case 2: $8 \operatorname{diam} \Gamma \leq r \leq \operatorname{diam} X$. We may find a point $x \in X$ such that $d(x, z)=$ $r / 4$. Since $\operatorname{diam} \Gamma \leq r / 8$ and $z \in \Gamma$, we see that

$$
\operatorname{dist}(x, \Gamma) \geq d(x, z)-\operatorname{diam} \Gamma \geq r / 8 .
$$

Hence $B(x, r / 8) \subseteq B(z, r) \backslash \Gamma$.

Case 3: $\operatorname{diam} \Gamma /(4 \Lambda) \leq r<8 \operatorname{diam} \Gamma$. In this case,

$$
\frac{r}{32 \Lambda}<\frac{\operatorname{diam} \Gamma}{4 \Lambda}
$$

Thus, Case 1 implies that there is a point $x \in X$ such that

$$
B\left(x, \frac{r}{32 \Lambda s}\right) \subseteq B\left(z, \frac{r}{32 \Lambda}\right) \backslash \Gamma \subseteq B(z, r) \backslash \Gamma .
$$

Remark 8.3. Theorem 8.2 is also true for weak-quasicircles; see [22].

We combine the results of this section with those of the previous sections in the following statement:

Corollary 8.4. Let $(X, d)$ be a doubling metric space that is homeomorphic to a domain in $\mathbb{S}^{2}$, has compact completion, and satisfies the ALLC condition. Then each component of the boundary $\partial X$ is a porous subset of $\bar{X}$, quantitatively.

Proof. By Lemma 2.5, the space $X$ is also LLC, quantitatively. Theorem 1.6 now implies that each component of the boundary $\partial X$ is a quasicircle, quantitatively. Thus Theorem 8.2 yields the desired result.

The following theorem states that, up to bi-Lipschitz equivalence, having Assouad dimension strictly less than 2 characterizes quasicircles in $\mathbb{S}^{2}$ among the class of all quasicircles [16]. 
Theorem 8.5 (Herron-Meyer). Let $\left(\Gamma, d_{\Gamma}\right)$ be a metric circle. The following statements are equivalent, quantitatively:

- $\Gamma$ is a quasicircle that is $(\alpha, C)$-homogeneous for some $1 \leq \alpha<2$ and $C \geq 1$.

- $\Gamma$ is bi-Lipschitz equivalent to a quasicircle in $\mathbb{S}^{2}$.

Remark 8.6. Combined with the classical theory of planar quasiconformal mappings, Theorem 8.5 has the following consequence, which we will use in the proof of our main result. Let $\Gamma$ be a quasicircle that is $(\alpha, C)$-homogeneous for some $1 \leq \alpha<2$ and $C \geq 1$. Then there is a domain $D_{\Gamma} \subseteq \mathbb{S}^{2}$ such that

(i) $D_{\Gamma}$ is Ahlfors 2-regular,

(ii) $D_{\Gamma}$ is quasisymmetrically equivalent to $\mathbb{D}^{2}$,

(iii) the boundary $\partial D_{\Gamma}$ is bi-Lipschitz equivalent to $\Gamma$.

\section{Gluing}

We now describe a process for gluing together metric spaces along bi-Lipschitz equivalent subsets. Parts of the basic construction may be found in [7], and related deeper results are included in [12].

For the remainder of this section, we let $I$ be a possibly uncountable index set, which we extend by a symbol 0 to create the index set $I_{0}=I \cup\{0\}$. We consider a collection $\left\{\left(X_{i}, d_{i}\right)\right\}_{i \in I_{0}}$ of compact metric spaces and a pairwise disjoint collection $\left\{E_{i}\right\}_{i \in I}$ of continua in $X_{0}$ such that there is a number $L \geq 1$ such that for each $i \in I$, there exists an $L$-bi-Lipschitz homeomorphism $f_{i}: E_{i} \rightarrow f_{i}\left(E_{i}\right) \subseteq X_{i}$.

\subsection{The basic gluing construction}

We first consider the disjoint union

$$
\widetilde{Z}=\coprod_{i \in I_{0}} X_{i}
$$

We then consider the set $Z$ obtained by gluing each space $X_{i}$ to $X_{0}$ via $f_{i}$, i.e., $Z$ is the quotient of $\widetilde{Z}$ by the equivalence relation $\sim$ generated by the condition that for all $i \in I$, if $x \in E_{i}$, then $x \sim f_{i}(x)$. The usual quotient map sending a point of $\widetilde{Z}$ to its equivalence class is denoted by $\pi$.

We wish to define a natural metric on $Z$. To do so, we define an auxiliary distance function for points $z, w \in \widetilde{Z}$ by

$$
\widetilde{d}(z, w)= \begin{cases}d_{i}(z, w) & z, w \in X_{i} \\ \infty & \text { otherwise }\end{cases}
$$

We now define a distance function $d$ on $Z$ by setting, for all equivalence classes $a, b \in Z$,

$$
d(a, b)=\inf \sum_{k=1}^{n} \widetilde{d}\left(z_{k}, z_{k}^{\prime}\right),
$$


where the infimum is taken over all sequences $\mathbf{z}=z_{1}, z_{1}^{\prime}, \ldots, z_{n}, z_{n}^{\prime}$ in $\widetilde{Z}$ such that $z_{1} \in a, z_{n}^{\prime} \in b$, and if $n>1$, then $\pi\left(z_{k}^{\prime}\right)=\pi\left(z_{k+1}\right)$ for all $i=1, \ldots, n-1$. We say that such a sequence $\mathbf{z}$ is an admissible sequence from $a$ to $b$ if the $\widetilde{d}$-length of $\mathbf{z}$,

$$
l_{\widetilde{d}}(\mathbf{z})=\sum_{k=1}^{n} \widetilde{d}\left(z_{k}, z_{k}^{\prime}\right),
$$

is finite, and if for any $k=1, \ldots, n-1$,

$$
z_{k}^{\prime} \neq z_{k+1}
$$

The triangle inequality implies that the infimum in the definition of $d(a, b)$ may be taken over all admissible sequences.

Proposition 9.1. The distance function $d$ is a metric on the set $Z$, and if points $a$ and $b$ of $Z$ have representatives $z_{a}$ and $z_{b}$ satisfying $\widetilde{d}\left(z_{a}, z_{b}\right)<\infty$, then

$$
\frac{\widetilde{d}\left(z_{a}, z_{b}\right)}{L} \leq d(a, b) \leq \widetilde{d}\left(z_{a}, z_{b}\right) .
$$

Proof. Let $a, b \in Z$. The definitions quickly imply that $d(a, b)=d(b, a)$, that $d(a, a)=0$, and that $d$ satisfies the triangle inequality.

Before showing that $d(a, b)=0$ implies that $a=b$, we prove (9.2). Let $z_{a}$ and $z_{b}$ be representatives of $a$ and $b$ respectively, such that $\widetilde{d}\left(z_{a}, z_{b}\right)<\infty$. The second inequality in (9.2) follows from the fact that $z_{a}, z_{b}$ is an admissible sequence connecting $a$ to $b$. Towards a proof of the first inequality, let $\mathbf{z}=z_{1}, z_{1}^{\prime}, \ldots, z_{n}, z_{n}^{\prime}$ be an admissible sequence connecting $a$ to $b$. We consider only the case that $z_{a}$ and $z_{b}$ are in $X_{0}$; the other cases are handled similarly. It suffices to assume that $z_{1}=z_{a}$ and $z_{n}^{\prime}=z_{b}$ and to show that $l_{\widetilde{d}}(\mathbf{z}) \geq \widetilde{d}\left(z_{a}, z_{b}\right) / L$. For each $k=1, \ldots, n$, let $i_{k} \in I_{0}$ be the index such that $z_{k}, z_{k}^{\prime} \in X_{i_{k}}$. Since $\mathbf{z}$ is admissible, we may assume that if $i_{k} \neq 0$, then $z_{k}, z_{k}^{\prime} \in f_{i_{k}}\left(E_{i_{k}}\right)$. Since each $f_{i_{k}}$ is an $L$-bi-Lipschitz mapping, the triangle inequality implies that

$$
\begin{aligned}
l_{\widetilde{d}}(\mathbf{z}) & =\sum_{k=1}^{n} d_{i_{k}}\left(z_{k}, z_{k}^{\prime}\right) \geq \sum_{\left\{k: i_{k}=0\right\}} d_{0}\left(z_{k}, z_{k}^{\prime}\right)+\sum_{\left\{k: i_{k} \neq 0\right\}} \frac{d_{0}\left(f_{i_{k}}^{-1}\left(z_{k}\right), f_{i_{k}}^{-1}\left(z_{k}^{\prime}\right)\right)}{L} \\
& \geq \frac{d_{0}\left(z_{a}, z_{b}\right)}{L}=\frac{\widetilde{d}\left(z_{a}, z_{b}\right)}{L},
\end{aligned}
$$

as desired.

Now, suppose that $d(a, b)=0$. If there are representatives $z_{a}$ and $z_{b}$ of $a$ and $b$ respectively that satisfy $\widetilde{d}\left(z_{a}, z_{b}\right)<\infty$, then $(9.2)$ shows that $z_{a}=z_{b}$, and hence $a=b$. Suppose no such representatives exist. Then we may assume without loss of generality that $a$ has a representative $z_{a} \in X_{i} \backslash f_{i}\left(E_{i}\right)$ for some $i \in I$, and that $b$ has no representative in $X_{i}$. Then any admissible sequence from $a$ to $b$ has $\widetilde{d}$-length at least $\operatorname{dist}_{d_{i}}\left(z_{a}, f_{i}\left(E_{i}\right)\right)$. Since $f_{i}\left(E_{i}\right)$ is compact, we conclude that $d(a, b)$ is positive, a contradiction. 
Remark 9.2. The metric $d$ defines a topology on $Z$, which we will refer to as the metric topology on $Z$. There is another natural topology on $Z$, which we will refer to as the quotient topology on $Z$. It is obtained as follows. First, we equip $\widetilde{Z}$ with the disjoint union topology, i.e., a set $A \subseteq \widetilde{Z}$ is open if and only for each $i \in I_{0}$, the set $A \cap X_{i}$ is open in $X_{i}$. Note that a $\widetilde{d}$-ball in $\widetilde{Z}$ of finite radius is open. Then we consider the quotient topology on $Z$ arising from the equivalence relation $\sim$, i.e., the maximal topology on $Z$ in which the standard projection map $\pi: \widetilde{Z} \rightarrow Z$ continuous. It is not hard to check that every open set in the metric topology on $Z$ is open in the quotient topology on $Z$. Moreover, if card $I<\infty$, then the topologies coincide. Simple examples show that the topologies may differ if this is not the case.

The following proposition states that away from the gluing sets, the space $Z$ is locally isometric to $\widetilde{Z}$.

Proposition 9.3. Let $r>0$, and suppose that $a \in Z$ satisfies

$$
\operatorname{dist}_{d}\left(a, \pi\left(\bigcup_{i \in I} E_{i}\right)\right) \geq 3 r .
$$

Then there is unique representative $z_{b}$ of each $b \in B_{d}(a, r)$, and $\pi^{-1}: B_{d}(a, r) \rightarrow$ $B_{\widetilde{d}}\left(z_{a}, r\right)$ is a well-defined bijective isometry.

Proof. Let $b$ and $c$ be points of $B_{d}(a, r)$. Then Proposition 9.1 and (9.3) imply that if $z_{b}$ and $z_{c}$ are representatives of $b$ and $c$ respectively, then

$$
\operatorname{dist}_{\widetilde{d}}\left(\left\{z_{b}, z_{c}\right\}, \bigcup_{i \in I}\left(f_{i}\left(E_{i}\right) \cup E_{i}\right)\right) \geq 2 r .
$$

This immediately implies that $b$ and $c$ have unique representatives $z_{b}$ and $z_{c}$, respectively. Since $d(b, c)<2 r$, there is admissible sequence $\mathbf{z}$ from $b$ to $c$ that has $\widetilde{d}$-length less than $2 r$. The conditions (9.1) and (9.4) now imply that $\mathbf{z}=z_{b}, z_{c}$, and hence that $d(b, c)=\widetilde{d}\left(z_{b}, z_{c}\right)$. A similar argument shows that $z_{b}$ and $z_{c}$ are in $B_{\widetilde{d}}\left(z_{a}, r\right)$. These facts together imply the desired statement.

\subsection{Preservation of the ALLC condition}

In this and the following subsection, we make the following assumption on the spaces in the collection $\left\{X_{i}\right\}_{i \in I}$ :

(A) there is a constant $C \geq 1$ such that $\operatorname{diam}_{d_{i}}\left(X_{i}\right) \leq C \operatorname{diam}_{d_{i}} f_{i}\left(E_{i}\right)$ for all $i \in I$. Heuristically, this assumption means that the spaces $\left(X_{i}, d_{i}\right)$ are "flat".

We now show that if each space in the collection $\left\{X_{i}\right\}_{i \in I_{0}}$ satisfies the ALLC condition with a uniform constant, then the glued space $(Z, d)$ also satisfies the ALLC condition, quantitatively.

Lemma 9.4. Suppose that there is a constant $\lambda \geq 1$ such that for each $i \in I_{0}$, the space $\left(X_{i}, d_{i}\right)$ is $\lambda$-ALLC. Then there is a quantity $\Lambda \geq 1$, depending only on the data, with the following property. Let $i \in I, a \in Z$, and $r>0$. Then at least one of the following two statements holds: 
(i) the annulus $A_{d}(a, r, 2 r)$ is contained in $\pi\left(X_{i}\right)$,

(ii) there is a point $b^{\prime} \in A_{d}(a, r / \Lambda, 2 \Lambda r) \cap \pi\left(E_{i}\right)$ such that for each point $b \in$ $A_{d}(a, r, 2 r) \cap \pi\left(X_{i}\right)$, there is a continuum $E$ containing $b$ and $b^{\prime}$ satisfying

$$
E \subseteq A_{d}(a, r / \Lambda, 2 \Lambda r) \cap \pi\left(X_{i}\right)
$$

Proof. We first assume that $a \notin \pi\left(X_{i}\right)$, and will show that the second statement above holds. We consider two subcases.

Case 1: $r>32 L C \lambda \operatorname{dist}_{d}\left(a, \pi\left(E_{i}\right)\right)$. Let $a^{\prime}$ be a point of $\pi\left(E_{i}\right)$ such that

$$
d\left(a, a^{\prime}\right)<\frac{r}{32 L C \lambda} .
$$

The triangle inequality implies that

$$
A_{d}(a, r, 2 r) \subseteq A_{d}\left(a^{\prime}, \frac{r}{2}, 3 r\right) .
$$

If $\operatorname{diam}_{d_{i}} f_{i}\left(E_{i}\right)<r /(2 C)$, then Proposition 9.1 and condition (A) show that $\pi\left(X_{i}\right) \subseteq B_{d}\left(a^{\prime}, \frac{r}{2}\right)$. By $(9.5)$, this now implies that $A_{d}(a, r, 2 r) \cap \pi\left(X_{i}\right)$ is empty and hence the claim is vacuously true. Thus we may assume that

$$
\operatorname{diam}_{d_{i}} f_{i}\left(E_{i}\right) \geq \frac{r}{2 C}
$$

Let $z_{a^{\prime}}$ be the representative of $a^{\prime}$ in $f_{i}\left(E_{i}\right)$. The connectedness of $f_{i}\left(E_{i}\right)$ and $(9.6)$ imply that there is a point $z_{b^{\prime}} \in f_{i}\left(E_{i}\right)$ such that

$$
d_{i}\left(z_{a^{\prime}}, z_{b^{\prime}}\right)=\frac{r}{8 C}
$$

Set $b^{\prime}=\pi\left(z_{b^{\prime}}\right)$. Let $b \in A_{d}(a, r, 2 r)$, and denote by $z_{b}$ the representative of $b \in X_{i}$. Proposition 9.1, the above equality, and (9.5) show that

$$
z_{b}, z_{b^{\prime}} \in A_{d_{i}}\left(z_{a^{\prime}}, \frac{r}{16 C}, 3 L r\right)
$$

The $\lambda$-ALLC condition in $X_{i}$ now provides a continuum $E^{\prime}$ containing $z_{b}$ and $z_{b}^{\prime}$ such that

$$
E^{\prime} \subseteq A_{d_{i}}\left(z_{a^{\prime}}, \frac{r}{16 C \lambda}, 3 L \lambda r\right)
$$

Proposition 9.1 shows that

$$
\pi\left(E^{\prime}\right) \subseteq A_{d}\left(a^{\prime}, \frac{r}{16 C L \lambda}, 3 L \lambda r\right) .
$$

The triangle inequality now shows that

$$
\pi\left(E^{\prime}\right) \subseteq A_{d}\left(a, \frac{r}{32 C L \lambda},(3 L \lambda+1) r\right) .
$$

Clearly $\pi\left(E^{\prime}\right)$ is a continuum containing $b$ and $b^{\prime}$. 
Case 2: $r \leq 32 L C \lambda \operatorname{dist}_{d}\left(a, \pi\left(E_{i}\right)\right)$. We may assume that there is a point $b_{0} \in$ $A_{d}(a, r, 2 r) \cap \pi\left(X_{i}\right)$, for otherwise statement (ii) above is vacuously true. The definition of an admissible chain shows that this implies the existence of a point $b^{\prime} \in \pi\left(E_{i}\right)$ satisfying $d\left(a, b^{\prime}\right)<2 r$ and $d\left(b, b^{\prime}\right)<2 r$. Given a point $b \in A_{d}(a, r, 2 r) \cap \pi\left(X_{i}\right)$, Proposition 9.1 shows that we may find representatives $z_{b}$ and $z_{b^{\prime}}$ in $X_{i}$ of $b$ and $b^{\prime}$ respectively such that $d_{i}\left(z_{b}, z_{b^{\prime}}\right)<2 L r$. By Lemma 2.5, the $\lambda$-ALLC condition in $X_{i}$ provides a continuum $E^{\prime} \subseteq B_{d_{i}}\left(z_{b}, 4 L \lambda r\right)$ that connects $b$ and $b^{\prime}$. Proposition 9.1 implies that

$$
\pi\left(E^{\prime}\right) \subseteq B_{d}(b, 4 L \lambda r) \subseteq B_{d}(a,(4 L \lambda+2) r) .
$$

The restriction that $r \leq 32 L C \lambda \operatorname{dist}_{d}\left(a, \pi\left(E_{i}\right)\right)$ implies that $B_{d}(a, r /(32 L C \lambda)$ does not intersect $\pi\left(E_{i}\right)$. The definition of an admissible sequence now shows that $B_{d}\left(a, r /(32 L C \lambda)\right.$ does not intersect $\pi\left(X_{i}\right)$. Thus $\pi\left(E^{\prime}\right)$ is a continuum containing $b$ and $b^{\prime}$ and satisfying

$$
\pi\left(E^{\prime}\right) \subseteq A_{d}\left(a, \frac{r}{32 L C \lambda},(4 L \lambda+2) r\right) .
$$

Now, we assume that $a \in \pi\left(X_{i}\right)$, that the first statement above does not hold, and that the second statement above is not trivially true. That is, we assume that $A_{d}(a, r, 2 r)$ intersects both $\pi\left(X_{i}\right)$ and $Z \backslash \pi\left(X_{i}\right)$.

We claim that $A_{d}(a, r /(4 L C), 2 r)$ intersects $\pi\left(E_{i}\right)$. Since $\pi\left(E_{i}\right)$ is connected, if this is not the case, then either $\pi\left(E_{i}\right) \subseteq \bar{B}_{d}(a, r /(4 L C))$ or $\pi\left(E_{i}\right) \subseteq Z \backslash B_{d}(a, 2 r)$. If the first possibility occurs, then condition (A) yields a contradiction with the assumptions that $A_{d}(a, r, 2 r)$ meets $\pi\left(X_{i}\right)$ and that $a \in \pi\left(X_{i}\right)$. If the second possibility occurs, then the assumption that $A_{d}(a, r, 2 r)$ meets $Z \backslash \pi\left(X_{i}\right)$ and the definition of admissible chain yield a contradiction.

Thus we may find a point $b^{\prime} \in A_{d}(a, r /(4 L C), 2 r) \cap \pi\left(E_{i}\right)$. That this point satisfies the requirements of the second statement of the lemma is left to the reader.

Lemma 9.5. Suppose that there is a constant $\lambda \geq 1$ such that for each $i \in I_{0}$, the space $\left(X_{i}, d_{i}\right)$ is $\lambda$-ALLC. There is a quantity $\Lambda \geq 1$, depending only on the data, with the following property. Let $a \in Z$ and $r>0$. If $u$ and $v$ are points in $A_{d}(a, r, 2 r) \cap \pi\left(X_{0}\right)$, then there is a continuum $E$ containing $u$ and $v$ satisfying

$$
E \subseteq A_{d}\left(a, \frac{r}{\Lambda}, 2 \Lambda r\right)
$$

Proof. We claim that $\Lambda=4 L \lambda+2$ fulfils the requirements of the lemma. If $a \in \pi\left(X_{0}\right)$, this follows from Proposition 9.1 and the $\lambda$-ALLC condition on $X_{0}$; the details are left to the reader. Hence we assume that $a \notin \pi\left(X_{0}\right)$, and set

$$
s=\frac{L \lambda+1}{2} .
$$

First, we consider the case that $r \leq s \operatorname{dist}_{d}\left(a, \pi\left(X_{0}\right)\right)$. Then $B_{d}(a, r / s)$ does not intersect $\pi\left(X_{0}\right)$, and so the existence of the desired continuum follows from Proposition 9.1 and Lemma 2.5; again, the details are left to the reader. 
Now suppose that $r>s \operatorname{dist}_{d}\left(a, \pi\left(X_{0}\right)\right)$. Then there is a point $a^{\prime} \in \pi\left(X_{0}\right)$ such that $d\left(a, a^{\prime}\right)=\operatorname{dist}_{d}\left(a, \pi\left(X_{0}\right)\right)$. The triangle inequality yields

$$
A_{d}(a, r, 2 r) \subseteq A_{d}\left(a^{\prime}, \frac{(s-1) r}{s}, \frac{(2 s+1) r}{s}\right) .
$$

Let $z_{u}, z_{v}$, and $z_{a^{\prime}}$ be representatives of $u, v, a^{\prime}$, respectively, that are contained in $X_{0}$. Proposition 9.1 and the $\lambda$-ALLC condition in $X_{0}$ imply that there is a continuum $E^{\prime} \subseteq X_{0}$ containing $z_{u}$ and $z_{v}$ satisfying

$$
E^{\prime} \subseteq A_{d_{0}}\left(z_{a^{\prime}}, \frac{(s-1) r}{\lambda s}, \frac{(2 s+1) L \lambda r}{s}\right) .
$$

Proposition 9.1, the triangle inequality, and the definition of $s$ now show that

$$
\pi\left(E^{\prime}\right) \subseteq A_{d}\left(a^{\prime}, \frac{(s-1) r}{L \lambda s}, \frac{(2 s+1) L \lambda r}{s}\right) \subseteq A_{d}\left(a, \frac{r}{2 L \lambda},(2 L \lambda+2) r\right),
$$

proving the claim in this case as well.

Theorem 9.6. Suppose that there is a constant $\lambda \geq 1$ such that for each $i \in I_{0}$, the space $\left(X_{i}, d_{i}\right)$ is $\lambda$-ALLC. Then $(Z, d)$ is $\Lambda$-ALLC, where $\Lambda \geq 1$ depends only on the data.

Proof. Let $a \in Z$ and $r>0$. We show that each pair of points $u, v \in A_{d}(a, r, 2 r)$ is contained in a continuum $E \subseteq Z$ satisfying

$$
E \subseteq A_{d}\left(a, \frac{r}{\Lambda}, 2 \Lambda r\right)
$$

where $\Lambda \geq 1$ is now defined to be the maximum of the quantities provided by Lemmas 9.4 and 9.5 .

Choose representatives $z_{u} \in X_{i_{u}}$ and $z_{v} \in X_{i_{v}}$ of $u$ and $v$, respectively. If $i_{u}=i_{v} \in I$, then Lemma 9.4 provides the desired continuum. If $i_{u}=i_{v}=0$, then Lemma 9.5 provides the desired continuum. If $i_{u} \neq i_{v}$ and neither are 0 , we employ Lemma 9.4. If the first possibility in Lemma 9.4 holds, the desired continuum is easily constructed using Proposition 9.1. If the second possibility holds, we are provided with continua $E_{u}$ and $E_{v}$ that connect $u$ and $v$ to points $u^{\prime} \in \pi\left(X_{0}\right)$ and $v^{\prime} \in \pi\left(X_{0}\right)$ respectively, where

$$
E_{u} \cup E_{v} \subseteq A_{d}(a, r / \Lambda, 2 \Lambda r) .
$$

If $i_{u}=0$, then we instead set $u^{\prime}=u$, and similarly define $v^{\prime}=v$ if $i_{v}=0$. Applying Lemma 9.5 to $u^{\prime}$ and $v^{\prime}$ and concatenating now produces the desired continuum.

\subsection{Preservation of Ahlfors regularity}

In this subsection only, we add to condition (A) two assumptions on the geometry of the base space $X_{0}$. First, we assume the uniform relative separation of the gluing sets:

(B) there is a constant $c>0$ such that $\triangle\left(E_{i}, E_{j}\right) \geq c$ for all $i \neq j \in I$.

We also assume, without loss of generality, that $c \leq 1$. 
Our final assumption corresponds to condition (2) in the statement of Theorem 1.4. Namely, for each integer $k \geq 0$, set

$$
n_{k}:=\sup \operatorname{card}\left\{i \in I: E_{i} \cap B_{d_{0}}(z, r) \neq \emptyset \text { and } 2^{-k}<\frac{\operatorname{diam}_{d_{0}}\left(E_{i}\right)}{r} \leq 2^{-k+1}\right\},
$$

where the supremum is taken over all $z \in X_{0}$ and $0<r \leq 2 \operatorname{diam}_{d_{0}} X_{0}$. We assume that there are numbers $Q>0$ and $1 \leq M \leq \infty$ such that

(C) $\sum_{k \in \mathbb{N}} n_{k}\left(2^{-k}\right)^{Q} \leq M$.

Theorem 9.7. Suppose that there is a constant $K \geq 1$ such that for each $i \in I_{0}$, the space $\left(X_{i}, d_{i}\right)$ is Ahlfors $Q$-regular with constant $K$. Then $(Z, d)$ is Ahlfors $Q$-regular, quantitatively.

Proof. As noted in the definition of Ahlfors regularity, it suffices to show that for $a \in Z$ and $0<r \leq 2 \operatorname{diam}_{d} Z$,

$$
\mathcal{H}_{(Z, d)}^{Q}\left(B_{d}(a, r)\right) \simeq r^{Q}
$$

First suppose that $a$ satisfies

$$
\operatorname{dist}_{d}\left(a, \pi\left(\bigcup_{i \in I} E_{i}\right)\right) \geq 3 r
$$

Then Proposition 9.3 implies that

$$
\mathcal{H}_{(Z, d)}^{Q}\left(B_{d}(a, r)\right)=\mathcal{H}_{(\widetilde{Z}, \widetilde{d})}^{Q}\left(B_{\widetilde{d}}\left(z_{a}, r\right)\right)=\mathcal{H}_{\left(X_{i}, d_{i}\right)}^{Q}\left(B_{d_{i}}\left(z_{a}, r\right)\right),
$$

where $z_{a} \in X_{i}$ is the unique representative of $a$. It also follows that $r$ can be no larger than twice the diameter of $\left(X_{i}, d_{i}\right)$. Since $\left(X_{i}, d_{i}\right)$ is Ahlfors $Q$-regular, the desired estimate follows.

Next we suppose that $a \in \pi\left(E_{i}\right)$ for some $i \in I$. Let $z_{a}^{0} \in E_{i}$ and $z_{a}=f_{i}\left(z_{a}^{0}\right) \in$ $f_{i}\left(E_{i}\right) \subseteq X_{i}$ be the representatives of $a$. Set $B_{0}=B_{d}(a, r) \cap \pi\left(X_{0}\right)$. Proposition 9.1 implies that $\left.\pi\right|_{X_{0}}$ is an $L$-bi-Lipschitz mapping, and that

$$
\pi\left(B_{\widetilde{d}}\left(z_{a}^{0}, r\right)\right) \subseteq B_{0} \subseteq \pi\left(B_{\widetilde{d}}\left(z_{a}^{0}, L r\right)\right)
$$

Note that by the triangle inequality, Proposition 9.1, and condition (A),

$$
\operatorname{diam}_{d} Z \simeq \operatorname{diam}_{d_{0}} X_{0}
$$

Hence, we may apply the Ahlfors $Q$-regularity of $\left(X_{0}, d_{0}\right)$ to see that

$$
\mathcal{H}_{(Z, d)}^{Q}\left(B_{0}\right) \simeq \mathcal{H}_{\left(X_{0}, d_{0}\right)}^{Q}\left(B_{d_{0}}\left(z_{a}^{0}, r\right)\right) \simeq r^{Q} .
$$

Thus

$$
\mathcal{H}_{(Z, d)}^{Q}\left(B_{d}(a, r)\right) \gtrsim r^{Q}
$$


We now work towards an upper bound for $\mathcal{H}_{(Z, d)}^{Q}\left(B_{d}(a, r)\right)$. For $j \in I$ set $B_{j}=B_{d}(a, r) \cap \pi\left(X_{j}\right)$, and let $J \subseteq I$ be the set of indices such that $B_{j} \neq \emptyset$. Furthermore, for $k \in \mathbb{Z}$, define

$$
J^{k}=\left\{j \in J: 2^{-k}<\frac{\operatorname{diam}_{d_{0}}\left(E_{j}\right)}{L c^{-1} r} \leq 2^{-k+1}\right\} .
$$

Now, we may write

$$
B_{d}(a, r)=B_{0} \bigcup \bigcup_{k \in \mathbb{Z}} \bigcup_{j \in J^{k}} B_{j} .
$$

By definition, $B_{j} \subseteq \pi\left(X_{j}\right)$ for any $j \in J$. As before, by Proposition 9.1, $\left.\pi\right|_{X_{j}}$ is an $L$-bi-Lipschitz mapping. Thus the Ahlfors $Q$-regularity of each $X_{j}$ and condition (A) imply that

$$
\mathcal{H}_{(Z, d)}^{Q}\left(B_{j}\right) \leq \mathcal{H}_{(Z, d)}^{Q}\left(\pi\left(X_{j}\right)\right) \simeq \mathcal{H}_{\left(X_{j}, d_{j}\right)}^{Q}\left(X_{j}\right) \simeq\left(\operatorname{diam}_{d_{0}} E_{j}\right)^{Q} .
$$

Fix $j \in J$. We claim that $E_{j} \cap B_{d_{0}}\left(z_{a}^{0}, L r\right) \neq \emptyset$. By definition, we may find a point $b \in \pi\left(X_{j}\right)$ and an admissible chain $\mathbf{z}$ from $a$ to $b$ of $\widetilde{d}$-length less than $r$. It follows from the definitions that there is an admissible subchain $\mathbf{z}^{\prime}$ connecting $a$ to a point $b^{\prime} \in \pi\left(E_{j}\right)$, and the $\widetilde{d}$-length of $\mathbf{z}^{\prime}$ is also less than $r$. Let $z_{b^{\prime}}^{0}$ be the representative of $b^{\prime}$ in $E_{j}$. By Proposition 9.1, it holds that

$$
d_{0}\left(z_{a}^{0}, z_{b^{\prime}}^{0}\right)<L r
$$

proving the claim. Since we have assumed that $c \leq 1$, the claim implies that card $J^{k} \leq n_{k}$ for each integer $k \geq 0$.

It now follows from condition (B) that there is at most one index $j \in J$ with the property that $\operatorname{diam}_{d_{0}} E_{j}>2 L c^{-1} r$, and hence

$$
\operatorname{card}\left(\bigcup_{k \leq-1} J^{k}\right) \leq 1
$$

Suppose $j_{0} \in J$ is an index with the above property, and let $a_{j_{0}}$ be a point of $B_{j_{0}}$. Then by the triangle inequality,

$$
B_{j_{0}} \subseteq B_{d}\left(a_{j_{0}}, 2 r\right) \cap \pi\left(X_{j_{0}}\right) .
$$

Proposition 9.1 implies that $\left.\pi\right|_{X_{j_{0}}}$ is an $L$-bi-Lipschitz mapping onto $\pi\left(X_{j_{0}}\right)$. Hence the Ahlfors $Q$-regularity of $X_{j_{0}}$ implies that

$$
\mathcal{H}_{(Z, d)}^{Q}\left(B_{j_{0}}\right) \leq \mathcal{H}_{(Z, d)}^{Q}\left(B_{d}\left(a_{j_{0}}, 2 r\right) \cap \pi\left(X_{j_{0}}\right)\right) \lesssim r^{Q} .
$$

Thus, inequalities (9.8), (9.9), and (9.10), along with condition (C), imply that

$$
\begin{aligned}
\mathcal{H}_{(Z, d)}^{Q}\left(B_{d}(a, r)\right) \leq \mathcal{H}_{(Z, d)}^{Q}\left(B_{0}\right)+\sum_{k \in \mathbb{Z}} \sum_{j \in J^{k}} \mathcal{H}^{Q}\left(B_{j}\right) \\
\quad \lesssim r^{Q}+\sum_{k=0}^{\infty} \sum_{j \in J^{k}} \operatorname{diam}_{d_{0}}\left(E_{j}\right)^{Q} \lesssim r^{Q}+r^{Q}\left(\sum_{k=0}^{\infty} \operatorname{card}\left(J^{k}\right)\left(2^{-k}\right)^{Q}\right) \lesssim r^{Q}
\end{aligned}
$$

as desired. Note that this upper bound is also valid when $r>2 \operatorname{diam}_{d} Z$. 
Finally, we consider the full case that

$$
\operatorname{dist}_{d}\left(a, \pi\left(\bigcup_{i \in I} E_{i}\right)\right)<3 r .
$$

We may find an index $i_{0} \in I$ and a point $b \in \pi\left(E_{i_{0}}\right)$ such that $d(a, b)<3 r$. Thus the triangle inequality and the previous case show that

$$
\mathcal{H}_{(Z, d)}^{Q}\left(B_{d}(a, r)\right) \leq \mathcal{H}_{(Z, d)}^{Q}\left(B_{d}(b, 4 r)\right) \lesssim r^{Q} .
$$

To get the desired lower bound, we consider two subcases. If

$$
\operatorname{dist}_{d}\left(a, \pi\left(\bigcup_{i \in I} E_{i}\right)\right)<r / 2,
$$

then, as above, we may find an index $i_{0} \in I$ and a point $b \in \pi\left(E_{i_{0}}\right)$ such that $d(a, b)<r / 2$. The triangle inequality and the previous case now show that

$$
\mathcal{H}_{(Z, d)}^{Q}\left(B_{d}(a, r)\right) \geq \mathcal{H}_{(Z, d)}^{Q}\left(B_{d}(b, r / 2)\right) \gtrsim r^{Q} .
$$

If (9.11) does not hold, then setting $r^{\prime}=r / 6$, we see that

$$
\operatorname{dist}_{d}\left(a, \pi\left(\bigcup_{i \in I} E_{i}\right)\right) \geq 3 r^{\prime}
$$

and we may apply the first case considered in the proof to conclude that

$$
\mathcal{H}_{(Z, d)}^{Q}\left(B_{d}(a, r)\right) \geq \mathcal{H}_{(Z, d)}^{Q}\left(B_{d}\left(a, r^{\prime}\right)\right) \gtrsim\left(r^{\prime}\right)^{Q} \simeq r^{Q},
$$

as desired.

\section{Putting it together}

In this section, we synthesize the results of the previous sections to produce a proof of our main result. We begin by setting up an induction.

Let $Y$ be a metric space. Given a subset $\mathcal{S}$ of $\mathcal{C}(Y)$, denote by cl $\mathcal{S}$ the topological closure of $\mathcal{S}$ in $\mathcal{C}(Y)$. Let $\mathcal{N}(Y) \subseteq \mathcal{C}(Y)$ denote the collection of nontrivial components of $\partial X$, and let $\mathcal{I}(Y)$ denote the points of $\mathcal{N}(Y)$ that are isolated points of $\mathcal{C}(Y)$.

Lemma 10.1. Let $(X, d)$ be a metric space, homeomorphic to a domain in $\mathbb{S}^{2}$, such that conditions (1)-(5) of Theorem 1.4 hold. Then $(X, d)$ bi-Lipschitzly embeds into a metric space $\left(Z, d_{Z}\right)$ that is homeomorphic to a domain in $\mathbb{S}^{2}$, satisfies conditions (1)-(5) of Theorem 1.4 quantitatively, and such that $\mathcal{C}(Z)$ is homeomorphic to $\mathcal{C}(X) \backslash \mathcal{I}(X)$.

Proof. We leave the verification of the quantitativeness of the statement to the reader, as it follows easily from the quantitativeness of the results proven thus far.

Denote $\mathcal{I}(X)=\left\{E_{i}\right\}_{i \in I}$. Since $(X, d)$ has compact completion, the index set $I$ has cardinality no larger than countably infinite. 
Fix $i \in I$. Since $X$ is Ahlfors 2-regular, it is doubling. Lemma 2.5 implies that $X$ is LLC. Hence, Theorem 1.6 and Corollary 8.4 imply that $E_{i}$ is a quasicircle that is porous in $\bar{X}$. Remark 8.6 provides an Ahlfors 2-regular quasidisk $D_{i} \subseteq \mathbb{S}^{2}$ with the property that there is a bi-Lipschitz map $f_{i}: E_{i} \rightarrow \partial D_{i}$. It is easily seen by using Proposition 10.10 in [14] that the ALLC property is preserved by quasisymmetric mappings. Hence, $D_{i}$ is ALLC. Note that none of the data of the conditions discussed in this paragraph depend on $i$.

We now apply the results of Section 9 . Let $X_{0}$ be the completion $\bar{X}$, and for each $i \in I$ set $X_{i}=\overline{D_{i}}$. We employ the bi-Lipschitz maps $f_{i}$ defined above as the gluing maps $f_{i}: E_{i} \rightarrow f_{i}\left(E_{i}\right) \subseteq X_{i}$. The conditions stated at the beginning of Section 9 are met by construction, and hence we may consider the resulting glued metric space $\left(Z, d_{Z}\right)$.

We first show that $Z$ is homeomorphic to a domain in $\mathbb{S}^{2}$. The proof is similar in spirit to that of Theorem 7.7. Denote

$$
\widetilde{X}=X \cup\left(\bigcup_{i \in I} E_{i}\right)
$$

By Theorem 7.7, there is a circle domain $\Omega^{\prime} \subseteq \mathbb{S}^{2}$ and a homeomorphism

$$
\widetilde{h}: \tilde{X} \rightarrow \Omega^{\prime} \cup\left(\bigcup_{F \in \mathcal{N}\left(\Omega^{\prime}\right)} F\right) .
$$

Denote the image of $\widetilde{h}$ by $\widetilde{\Omega^{\prime}}$. Since $\Omega^{\prime}$ is a circle domain, for each $i \in I$, we may write $\widetilde{h}\left(E_{i}\right)=S_{\mathbb{S}^{2}}\left(p_{i}, r_{i}\right)$, where $p_{i} \in \mathbb{S}^{2}$ and $r_{i}>0$. Since $\widetilde{h}$ induces a homeomorphism from $\mathcal{C}(X)$ to $\mathcal{C}\left(\widetilde{\Omega^{\prime}}\right)$, for each $i \in I$ there is a number $\epsilon_{i}>0$ such that

$$
\bar{B}_{\mathbb{S}^{2}}\left(p_{i}, r_{i}+\epsilon_{i}\right) \cap \partial \Omega^{\prime}=S_{\mathbb{S}^{2}}\left(p_{i}, r_{i}\right)
$$

and such that the resulting collection $\left\{\bar{B}_{\mathbb{S}^{2}}\left(p_{i}, r_{i}+\epsilon_{i}\right)\right\}_{i \in I}$ is pairwise disjoint. Moreover, the set

$$
\Psi=\Omega^{\prime} \cup\left(\bigcup_{i \in I} \bar{B}_{\mathbb{S}^{2}}\left(p_{i}, r_{i}\right)\right)
$$

is a domain in $\mathbb{S}^{2}$.

Proposition 9.1 implies that for each $i \in I_{0}$, there is a bi-Lipschitz homeomorphism $\iota_{i}: X_{i} \rightarrow \pi\left(X_{i}\right)$. Fix $i \in I$. There is a homeomorphism $g_{i}: X_{i} \rightarrow \bar{B}_{\mathbb{S}^{2}}\left(p_{i}, r_{i}\right)$. By Lemma 7.6, there is a homeomorphism $\Phi_{i}$ of $\bar{A}_{\mathbb{S}^{2}}\left(p_{i}, r_{i}, r_{i}+\epsilon_{i}\right)$ to itself such that $\Phi_{i}$ coincides with the identity on $S_{\mathbb{S}^{2}}\left(p_{i}, r_{i}+\epsilon_{i}\right)$ and coincides with $g_{i} \circ \iota_{i}^{-1} \circ \iota_{0} \circ \widetilde{h}^{-1}$ on $S_{\mathbb{S}^{2}}\left(p_{i}, r_{i}\right)$. Now, the map $H: Z \rightarrow \Psi$ defined by

$$
H(a)= \begin{cases}\widetilde{h} \circ \iota_{0}^{-1}(a) & a \in \pi\left(X_{0}\right) \backslash\left(\bigcup_{i \in I} \iota_{0} \circ \widetilde{h}^{-1}\left(\bar{B}_{\mathbb{S}^{2}}\left(p_{i}, r_{i}+\epsilon_{i}\right)\right)\right), \\ \Phi_{i} \circ \widetilde{h} \circ \iota_{0}^{-1}(a) & a \in \iota_{0} \circ \widetilde{h}^{-1}\left(\bar{A}_{\mathbb{S}^{2}}\left(p_{i}, r_{i}, r_{i}+\epsilon_{i}\right)\right), \\ g_{i} \circ \iota_{i}^{-1}(a) & a \in \pi\left(X_{i}\right),\end{cases}
$$

is the desired homeomorphism. See Figure 5.

We now verify that $Z$ satisfies conditions (1)-(5) of Theorem 1.4. Condition (A), which was imposed at the beginning of Subsection 9.2, is equivalent to the assertion that $\operatorname{diam} D_{i} \lesssim \operatorname{diam} \partial D_{i}$, which follows from the fact that $D_{i}$ is a 


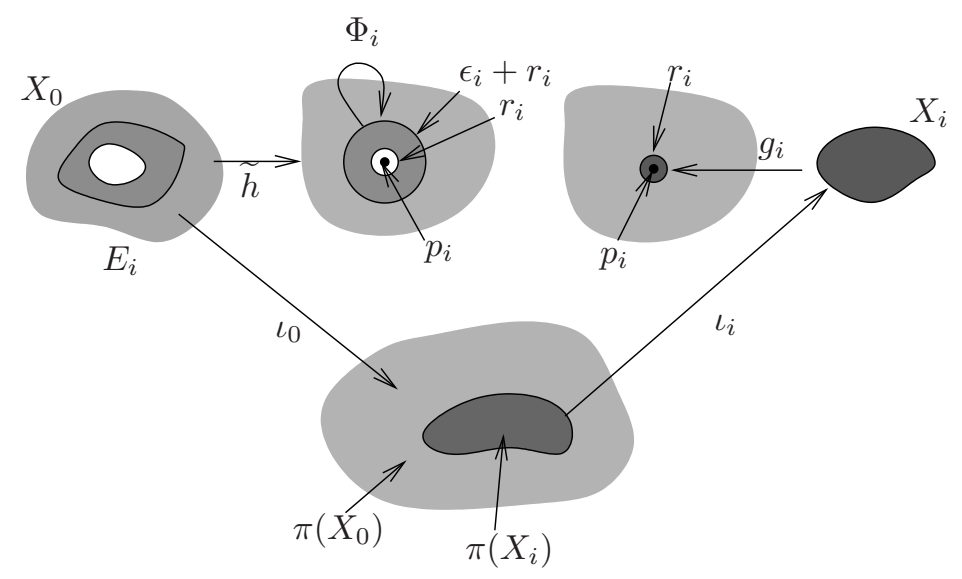

FiguRE 5. Filling in holes.

quasidisk or from the fact that $D_{i}$ is planar. Conditions (B) and (C), which were imposed at the beginning of Subsection 9.3, follow from the assumptions (5) and (2) in the statement of Theorem 1.4.

Hence, Theorems 9.6 and 9.7 imply that $\left(Z, d_{Z}\right)$ is ALLC and Ahlfors 2-regular. That $Z$ satisfies the remaining conditions $(2),(3),(5)$ of Theorem 1.4 and that $\mathcal{C}(Z)$ is homeomorphic to $\mathcal{C}(X) \backslash \mathcal{I}(X)$ follow from the construction and Proposition 9.1; we leave the details to the reader.

Proof of Theorem 1.4. The necessity of conditions (3)-(5) follows easily from the basic properties of quasisymmetric mappings and Proposition 2.7.

Now, let $(X, d)$ be a metric space, homeomorphic to a domain in $\mathbb{S}^{2}$, such that the closure of the non-isolated components of $\mathcal{C}(X)$ is countable and has finite rank, and such that conditions (1)-(5) hold. We will show that $(X, d)$ is quasisymmetrically equivalent to a circle domain whose collection of boundary components are uniformly relatively separated. Again, we leave the issue of quantitativeness to the reader.

We first reduce to the case that $\operatorname{cl} \mathcal{N}(X)=\mathcal{C}(X)$. Let $\mathcal{T}=\mathcal{C}(X) \backslash \operatorname{cl} \mathcal{N}(X)$. Consider the subspace $\widetilde{X}$ of $\bar{X}$ defined by

$$
\widetilde{X}=X \cup\left(\bigcup_{E \in \mathcal{T}} E\right)
$$

Let $h: \bar{X} \rightarrow \mathbb{S}^{2}$ be the continuous surjection provided by Corollary 4.6. Since each $E \in \mathcal{T}$ is trivial, Corollary 4.6 implies that the map $\left.h\right|_{\widetilde{X}}$ is a homeomorphism. Moreover, $h$ induces a homeomorphism of $\mathcal{C}(X)$ onto the totally disconnected set $\mathbb{S}^{2} \backslash h(X)$. Hence, the set $\{h(E)\}_{E \in \mathcal{T}}$ is open in $\mathbb{S}^{2} \backslash h(X)$. It follows that the image $h(\widetilde{X})$ is a domain in $\mathbb{S}^{2}$. Remark 2.2 and Proposition 2.6 imply that $\widetilde{X}$ is Ahlfors 2-regular and ALLC, quantitatively. Moreover, the space $\widetilde{X}$ clearly 
satisfies the remaining assumptions of Theorem 1.4, since $\mathcal{C}(\tilde{X})=\mathcal{C}(X) \backslash \mathcal{T}$ and hence $\operatorname{cl} \mathcal{N}(\tilde{X})=\operatorname{cl} \mathcal{N}(X)=\mathcal{C}(\tilde{X})$.

Thus, if Theorem 1.4 is valid for spaces such that the nontrivial boundary components are dense in the space of all boundary components, then applying the theorem to $\widetilde{X}$ and restricting the resulting quasisymmetric mapping to $X$ proves the theorem for $X$.

We now assume without loss of generality that $\operatorname{cl} \mathcal{N}(X)=\mathcal{C}(X)$. Our assumptions now imply that $\mathcal{C}(X)$ is countable and has finite rank, and we proceed by induction on the rank. If the rank of $\mathcal{C}(X)$ is 0 , then every boundary component is isolated. By Lemma 10.1, there is a bi-Lipschitz embedding $\iota: X \hookrightarrow Z$ where $\left(Z, d_{Z}\right)$ is complete, homeomorphic to a domain in $\mathbb{S}^{2}$, and satisfies conditions (1)-(5). Condition (3) implies that $Z$ is compact, and hence homeomorphic to $\mathbb{S}^{2}$. Lemma 2.5 implies that $Z$ is LLC. Bonk and Kleiner's uniformization result, Theorem 1.1, now provides a quasisymmetric homeomorphism $f: Z \rightarrow \mathbb{S}^{2}$. By Proposition 9.1, there is a bi-Lipschitz embedding $\iota: X \hookrightarrow Z$. The composition $f \circ \iota$ is a quasisymmetric homeomorphism onto its image $\Omega:=f \circ \iota(X)$. This mapping extends to a quasisymmetric homeomorphism of $\bar{X}$ onto $\bar{\Omega}$ (Proposition 10.10 in [14]). As discussed in the proof of Lemma 10.1, each of the components $\Gamma_{1}, \ldots, \Gamma_{N}$ of $\partial X$ is a quasicircle. Hence $\partial \Omega$ consists of finitely many quasicircles $\left\{f \circ \iota\left(\Gamma_{1}\right), \ldots, f \circ \iota\left(\Gamma_{N}\right)\right\}$, and, by Remark 2.1,

$$
\min _{i \neq j \in\{1, \ldots, N\}} \triangle\left(f \circ \iota\left(\Gamma_{i}\right), f \circ \iota\left(\Gamma_{j}\right)\right) \simeq \min _{i \neq j \in\{1, \ldots, N\}} \triangle\left(\Gamma_{i}, \Gamma_{j}\right) .
$$

Thus Bonk's uniformization result in $\mathbb{S}^{2}$, Theorem 1.7, provides a quasisymmetric homeomorphism $g: \mathbb{S}^{2} \rightarrow \mathbb{S}^{2}$ with the property that $g \circ f \circ \iota(X)$ is a circle domain. Again, the relative separation of the boundary components of this domain is controlled by Remark 2.1. This completes the proof in the case that the rank of $\mathcal{C}(X)$ is 0 .

We now assume that the desired statement is true in the case that the rank of $\mathcal{C}(X)$ is an integer $k \geq 1$, and suppose that the rank of $\mathcal{C}(X)$ is $k+1$. Theorem 10.1 now states that $X$ bi-Lipschitzly embeds into a metric space $\left(Z, d_{Z}\right)$ that is homeomorphic to a domain in the sphere, satisfies conditions (1)-(5), and such that $\mathcal{C}(Z)$ has rank $k$. By induction, $Z$ is quasisymmetrically equivalent to a circle domain. The remainder of the proof proceeds as in the base case.

Proof of Theorem 1.3. This follows from Proposition 2.8 and Theorem 1.4, after noting that the minimal relative separation of components of the boundary is controlled by the ratio of the minimal distance between components of the boundary to the diameter of the space.

Remark 10.2. We have defined a circle domain to be a subset of $\mathbb{S}^{2}$; one may also consider circle domains in $\mathbb{R}^{2}$, which need not have compact completion. An analogous version of Theorem 1.4 for such domains can easily be derived from Theorem 1.4 and the techniques of Section 6 in [27]. 


\section{An example}

In this section, we prove Theorem 1.5.

The desired space $(X, d)$ is obtained as follows. First we define a sequence of multiply connected domains $\left(Q_{n}\right)$. Let $Q_{0}$ denote the open unit square $(0,1) \times(0,1)$ in the plane. Let $Q_{1}$ be the domain obtained by removing the vertical line segment $\{1 / 2\} \times[1 / 4,3 / 4]$ from $Q_{0}$. We define $Q_{n+1}$ by subdividing $Q_{n}$ into $2^{n} \times 2^{n}$ dyadic subsquares of equal size in the obvious way, and replacing each square in the subdivision by a copy of $Q_{1}$ that has been scaled by $1 / 2^{n}$. See Figure 6 for $Q_{1}, Q_{2}$, and $Q_{3}$.
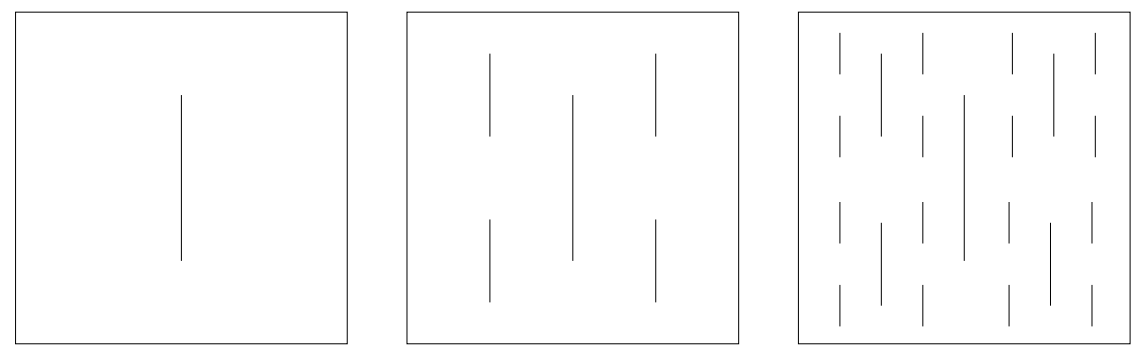

Figure 6. The domains $Q_{1}, Q_{2}$ and $Q_{3}$.

We denote by $\bar{Q}_{n}$ the completion of $Q_{n}$ in the path metric $d_{Q_{n}}$ induced from the plane. The induced metric on the completion is denoted by $d_{\bar{Q}_{n}}$. The boundary components of $Q_{n}$ give rise to the metric boundary components of $\left(Q_{n}, d_{Q_{n}}\right)$. The components of the metric boundary of $\left(Q_{n}, d_{Q_{n}}\right)$ that correspond to the boundary components of $Q_{n}$ other than the outer boundary will be called the slits of $\bar{Q}_{n}$.

Next we define a planar domain $Q$ inductively as follows. We start with $Q_{1}$ and replace the left-lower subsquare $(0,1 / 2) \times(0,1 / 2)$ by a copy of $Q_{2}$ that has been scaled by a factor of $1 / 2$. The resulting domain is denoted by $R_{1}$. Then we replace $R_{1} \cap((0,1 / 4) \times(0,1 / 4))$ by a copy of $Q_{3}$ that has been scaled by a factor of $1 / 4$. The resulting domain is denoted by $R_{2}$. We continue in this fashion and at the $n$-th step we replace $R_{n-1} \cap\left(\left(0,1 / 2^{n}\right) \times\left(0,1 / 2^{n}\right)\right)$ by a copy of $Q_{n+1}$ that has been scaled by a factor of $1 / 2^{n}$. The resulting domain is denoted by $R_{n}$. The countably connected domain that results after infinitely many such replacements is denoted by $Q$, see Figure 7 .

The desired metric space $(X, d)$ is the domain $Q$ endowed with the path metric $d_{Q}$ induced from the plane. We denote by $\pi_{\bar{X}}$ the projection of $\bar{X}$ onto $\bar{Q}_{0}$, i.e., the map that identifies the points in $\bar{X}$ that correspond to the same point of $\bar{Q}_{0}$. The map $\pi_{\bar{X}}$ is clearly 1-Lipschitz since the path metric on $Q$ dominates the Euclidean metric.

Alternatively, the space $\bar{X}$ can be defined as an inverse limit of the sequence of metric spaces $\left(\bar{R}_{n}, p_{m n}\right), m \leq n$. Here $\bar{R}_{n}$ is the completion of $R_{n}$ in the path metric induced from the plane and $p_{m n}$ is the projection of $\bar{R}_{n}$ onto $\bar{R}_{m}$ that identifies the points of $\bar{R}_{n}$ that correspond to the same point of $\bar{R}_{m}$. The map $\pi_{\bar{X}}$ 


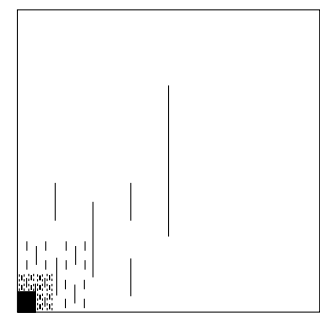

Figure 7. The domain $Q$.

is then the natural projection of $\bar{X}$ onto $\bar{Q}_{0}$. The extended metric $d$ on $\bar{X}$ now satisfies the equation

$$
d(p, q)=\lim d_{\bar{R}_{n}}\left(p_{n}, q_{n}\right),
$$

where $\left\{p_{n} \in \overline{R_{n}}\right\}$ and $\left\{q_{n} \in \overline{R_{n}}\right\}$ are sequences corresponding to $p$ and $q$ in the inverse limit system.

To establish the desired properties of the space $X$, we consider the slit carpet $S_{2}$ that has been studied in [21]. The space $S_{2}$ is the inverse limit of the system $\left(\bar{Q}_{n}, \pi_{m n}\right), m \leq n$, where $\pi_{m n}$ is the projection of $\bar{Q}_{n}$ onto $\bar{Q}_{m}$ that identifies the points on the slits of $\bar{Q}_{n}$ that correspond to the same point of $\bar{Q}_{m}$. See Figure 8 .

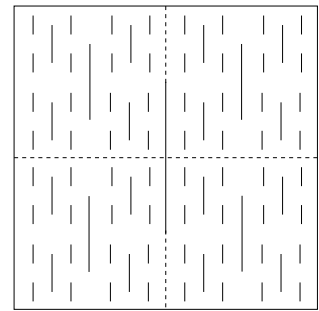

Figure 8 . The slit carpet $S_{2}$.

We make $S_{2}$ into a metric space by endowing it with the metric

$$
d_{S_{2}}(p, q)=\lim d_{\bar{Q}_{n}}\left(p_{n}, q_{n}\right),
$$

where $\left\{p_{n} \in \overline{Q_{n}}\right\}$ and $\left\{q_{n} \in \overline{Q_{n}}\right\}$ are sequences corresponding to $p$ and $q$ in the inverse limit system. The space $S_{2}$ is a geodesic metric space which is a metric Sierpiński carpet, i.e., a metric space homeomorphic to the well-known standard Sierpiński carpet, see Lemma 2.1 in [21]. The inverse limits of slits of $\bar{Q}_{n}$ form the family of peripheral circles of $S_{2}$, i.e., embedded simple closed curves whose removal does not separate $S_{2}$. The natural projection $\pi_{S_{2}}$ of $S_{2}$ onto $\bar{Q}_{0}$ factors through the projection $\pi_{S_{2} \rightarrow \bar{X}}$ of $S_{2}$ onto $\bar{X}$, i.e.,

$$
\pi_{S_{2}}=\pi_{\bar{X}} \circ \pi_{S_{2} \rightarrow \bar{X}} .
$$

The projection $\pi_{S_{2} \rightarrow \bar{X}}$ is clearly 1-Lipschitz. 
Lemma 11.1. There exists a constant $c>0$ such that for every $p \in \bar{X}$ and every $0 \leq r<2 \operatorname{diam}(X)$, there exists $q \in \bar{Q}_{0}$ with

$$
B_{\bar{Q}_{0}}(q, c \cdot r) \subseteq \pi_{\bar{X}}\left(B_{\bar{X}}(p, r)\right) \subseteq B_{\bar{Q}_{0}}\left(\pi_{\bar{X}}(p), r\right) .
$$

Proof. Fix $p \in \bar{X}$ and $0<r<2 \operatorname{diam}(X)$. The second inclusion follows since $\pi_{\bar{X}}$ is 1-Lipschitz. To show the first inclusion, we use the corresponding property of $S_{2}$ proved in [21]. Since $\pi_{S_{2} \rightarrow \bar{X}}$ is 1-Lipschitz, for any $p^{\prime} \in \pi_{S_{2} \rightarrow \bar{X}}^{-1}(p)$ we have

$$
\pi_{S_{2} \rightarrow \bar{X}}\left(B_{S_{2}}\left(p^{\prime}, r\right)\right) \subseteq B_{\bar{X}}(p, r) .
$$

By Lemma 2.2 in [21], there exists $c>0$ such that for any $p^{\prime}$ as above, there is $q \in \bar{Q}_{0}$ with

$$
B \bar{Q}_{0}(q, c \cdot r) \subseteq \pi_{S_{2}}\left(B_{S_{2}}\left(p^{\prime}, r\right)\right) .
$$

Combining these inclusions with the factorization of $\pi_{S_{2}}$ yields the desired inclusion.

The following lemma implies that the Lipschitz map $\pi_{\bar{X}}$ is David-Semmes regular, see Definition 12.1 in [9].

Lemma 11.2. There exists $C \geq 1$ such that for every $q \in \bar{Q}_{0}$ and $r>0$, the preimage $\pi_{\bar{X}}^{-1}(B(q, r))$ can be covered by at most $C$ balls in $\bar{X}$ of radii at most $C \cdot r$.

Proof. By Lemma 2.3 in [21], there exists $C \geq 1$ such that $\pi_{S_{2}}^{-1}(B(q, r))$ can be covered by at most $C$ balls with radii at most $C \cdot r$. If $\left(B\left(p_{i}^{\prime}, r_{i}\right)\right)$ is a family of such balls in $S_{2}$, then $\left(B\left(\pi_{S_{2} \rightarrow \bar{X}}\left(p_{i}^{\prime}\right), r_{i}\right)\right)$ is the desired family in $\bar{X}$.

Lemma 11.3. The metric space $X$ is homeomorphic to the planar domain $Q$ and satisfies conditions (1) and (3)-(5) of Theorem 1.4. Moreover, the rank of $\partial X$ is 1 .

Proof. The first assertion of the lemma follows from the fact that $Q$ is locally geodesic. We leave it to the reader to verify that each nontrivial component of $\partial X$ is a scaled copy of $\mathcal{S}^{1}$, that the collection of components of $\partial X$ is uniformly relatively separated, and that $\mathcal{C}(X)$ has a single limit point, which implies that the rank of $\partial X$ is 1 . The completion $\bar{X}$ is compact since $S_{2}$ is compact and $\pi_{S_{2} \rightarrow \bar{X}}$ is continuous.

We now establish the Alhfors 2-regularity of $X$. Since the boundary of $X$ has zero 2-measure, it is enough to show Ahlfors 2-regularity of the completion $\bar{X}$. Let $B_{\bar{X}}(p, r)$ be any ball with $0<r<2 \operatorname{diam}(\bar{X})$. Then, since $\pi_{\bar{X}}$ is 1 -Lipschitz,

$$
\mathcal{H}^{2}\left(B_{\bar{X}}(p, r)\right) \geq \mathcal{H}^{2}\left(\pi_{\bar{X}}\left(B_{\bar{X}}(p, r)\right)\right),
$$

and by the first inclusion in Lemma 11.1, the right-hand side is at least $r^{2} / C$ for some $C \geq 1$.

For the other inequality, we observe that, by Lemma 11.2, every cover of $\pi_{\bar{X}}\left(B_{\bar{X}}(p, r)\right)$ by open balls $\tilde{B}_{i}$ of radii $\tilde{r}_{i}$ at most some $\delta>0$ induces a cover of $B_{\bar{X}}(p, r)$ by balls $B_{j}$ of radii $r_{j}$ at most $C \cdot \delta$ with

$$
\sum_{j} r_{j}^{2} \leq C^{3} \sum_{i} \tilde{r}_{i}^{2}
$$


Since $\pi_{\bar{X}}\left(B_{\bar{X}}(p, r)\right)$ is contained in the Euclidean ball of radius $r$, the Ahlfors regularity now follows.

We will check the ALLC condition in several steps. We first check the $\mathrm{LLC}_{1}$ condition. Let $B_{X}(p, r)$ be an arbitrary ball and let $x, y \in B_{X}(p, r)$. Since $X$ is endowed with the path metric induced from the plane and $d(x, y)<2 r$, there is a curve $\gamma$ in $X$ that connects $x$ and $y$ and such that its length is at most $2 r$. Thus $E=\gamma$ is the desired continuum contained in $B(p, 3 r)$, i.e., $X$ satisfies the $3-\mathrm{LLC}_{1}$ condition.

Now we show that $\bar{X}$ satisfies the $\mathrm{LLC}_{2}$ condition. Let $B(p, r)$ be any ball in $\bar{X}$ and let $x, y \in \bar{X} \backslash B(p, r)$. We may assume that $r \leq 1$. Let $v_{x}$ denote a continuum in $\bar{X}$ that contains $x$ and projects by $\pi_{\bar{X}}$ one-to-one onto a closed vertical interval $I_{x}$ that satisfies the following properties. The end points of $I_{x}$ are $\pi_{\bar{X}}(x)$ and $\pi_{\bar{X}}\left(x^{\prime}\right)$ for some $x^{\prime} \in v_{x}$ with $\pi_{\bar{X}}\left(x^{\prime}\right)$ contained in the boundary of $Q_{0}$, so that the length of $I_{x}$ is not larger than the Euclidean distance from $\pi_{\bar{X}}(p)$ to the horizontal side of the boundary of $Q_{0}$ that contains $\pi_{\bar{X}}\left(x^{\prime}\right)$. We define $v_{y}$ and $y^{\prime}$ similarly. It follows from the choice of $v_{x}$ and $v_{y}$ that the distance from $p$ to $v_{x}$, respectively $v_{y}$, is at least $r / 2$. Indeed, suppose the distance from $p$ to, say, $v_{x}$ is less than $r / 2$. Then there exists $x^{\prime \prime} \in v_{x}$ so that $d_{\bar{X}}\left(p, x^{\prime \prime}\right)<r / 2$. Since $d_{\bar{X}}$ is the induced metric on the completion of $\left(Q, d_{Q}\right)$ and $d_{Q}$ is the path metric induced from the plane, $d_{\bar{X}}\left(x, x^{\prime \prime}\right)$ equals the Euclidean distance between $\pi_{\bar{X}}(x)$ and $p i_{\bar{X}}\left(x^{\prime \prime}\right)$, and $d_{\bar{X}}\left(p, x^{\prime \prime}\right)$ is at least the Euclidean distance between $\pi_{\bar{X}}(p)$ and $\pi_{\bar{X}}\left(x^{\prime \prime}\right)$. From the choice of $v_{x}$ we conclude that $d_{\bar{X}}\left(x, x^{\prime \prime}\right) \leq d_{\bar{X}}\left(p, x^{\prime \prime}\right)$. The triangle inequality yields a contradiction.

The points $x^{\prime}$ and $y^{\prime}$ are contained in some closed horizontal intervals $h_{x}$ and $h_{y}$ respectively, on the outer boundary of $X$ (i.e., the metric boundary component of $\left(Q, d_{Q}\right)$ that corresponds to the boundary of $\left.Q_{0}\right)$ whose lengths are $r / 4$. The distances from $h_{x}$ and $h_{y}$ to $p$ are then at least $r / 4$. If $v_{x} \cup v_{y} \cup h_{x} \cup h_{y}$ is connected, we are done. Otherwise, let $l$ and $l^{\prime}$ denote the two complementary components of $h_{x} \cup h_{y}$ in the outer boundary of $X$. We claim that at least one of $l$ or $l^{\prime}$ is at a distance at least $r / 8$ from $p$. Indeed, the sum of the distances from $p$ to $l$ and $l^{\prime}$ must be at least $r / 4$ because the length of every curve in $\bar{Q}_{0}$ separating $\pi_{\bar{X}}\left(v_{x} \cup h_{x}\right)$ from $\pi_{\bar{X}}\left(v_{y} \cup h_{y}\right)$ must be at least $r / 4$. Thus either $v_{x} \cup v_{y} \cup h_{x} \cup h_{y} \cup l$ or $v_{x} \cup v_{y} \cup h_{x} \cup h_{y} \cup l^{\prime}$ is the desired continuum $E$ in $\bar{X}$ in the LLC $_{2}$ condition with $\lambda=8$.

The next step is to establish the ALLC property for $\bar{X}$. Let $A_{\bar{X}}(p, r)$ denote $\bar{B}_{\bar{X}}(p, 2 r) \backslash B_{\bar{X}}(p, r)$ and let $x, y \in A(p, r)$. We may assume that $r \leq 1$. If $1 / 2 \leq$ $r \leq 1$, then the continuum $E$ found in the proof of the $\mathrm{LLC}_{2}$ condition works to conclude ALLC in this case, because the diameter of $E$ is at most 2 and thus it is at most $4 r$. If $0<r<1 / 2$, the proof of the existence of a desired continuum follows the lines similar to those in the proof of $\mathrm{LLC}_{2}$, but we first need to localize that argument. Indeed, first we can find a unique $n \in \mathbb{N}$ such that $1 / 2 \leq 2^{n} r<1$. Without loss of generality we may assume that $n$ is at least three. We consider the dyadic subdivision $\mathcal{D}$ of $Q_{0}$ into squares of side length $4 / 2^{n}$. Let $s$ be the interior of a square in this subdivision. We denote by $s_{\bar{X}}$ the preimage of $s$ under $\pi_{\bar{X}}$. Let also $\partial s_{\bar{X}}$ denote the metric boundary of $s_{\bar{X}}$, i.e., the closure of $s_{\bar{X}}$ in $\bar{X}$ less $s_{\bar{X}}$. 
Note that from the definitions of $\bar{X}$ and $\pi_{\bar{X}}$ it follows that $\partial s_{\bar{X}}$ is the union of four closed arcs, each isometric via $\pi_{\bar{X}}$ to a side of the boundary of $s$.

From the choice of $n$ and the fact that $\pi_{\bar{X}}$ is 1-Lipschitz, it follows that the projection $\pi_{\bar{X}}\left(\bar{B}_{\bar{X}}(p, 2 r)\right)$ can be covered by four squares from $\mathcal{D}$. Moreover, they can be chosen to be the first generation dyadic subsquares of a single, not necessarily dyadic, square of side length $8 / 2^{n}$. Let $\mathcal{F}$ be the family of the interiors of these four squares $s \in \mathcal{F}$. and let $K$ denote the closure in $\bar{X}$ of

$$
\cup_{s \in \mathcal{F}}\left(s_{\bar{X}}\right) .
$$

The set $K$ is compact and it contains $\bar{B}_{\bar{X}}(p, 2 r)$. The contour of $K$, denoted $c(K)$, is the closure in $\bar{X}$ of the set of all points $q$ such that $q$ belongs to $\partial s_{\bar{X}}$ for a unique $s \in \mathcal{F}$. The contour $c(K)$ is thus a union of closed arcs each of which is isometric to a horizontal or vertical side of the boundary of $s$ for some $s \in \mathcal{F}$. Since elements of $\mathcal{F}$ are interiors of the first generation dyadic subsquares of a single square, it is easy to see that $c(K)$ is connected, and thus it is a continuum. Considering various combinatorial possibilities for $c(K)$ one can easily conclude that $c(K)$ does not have global cut points. The rest of the proof of the ALLC property for $\bar{X}$ follows essentially the same lines as the proof of the $\mathrm{LLC}_{2}$ condition where the boundary of $Q_{0}$ should be replaced by $c(K)$. The diameter of the resulting continuum $E$ is at most $16 / 2^{n} \leq 32 r$.

Finally, given a point $p \in X$, a radius $r>0$, and points $x, y \in A_{X}(p, r, 2 r)$, we modify the above continuum $E$ to obtain one in $X$ as follows. If $E$ does not pass through the point $p_{0}$ in $\bar{X}$ that projects to $(0,0)$, then it has a neighborhood that intersects only finitely many boundary components of $X$, and thus $E$ can be modified slightly so that these boundary components are avoided. If $E$ does pass though $p_{0}$, then $p \neq p_{0}$, and we can first modify $E$ in an arbitrarily small neighborhood of $p_{0}$ to avoid this point and then apply the above.

If $(X, d)$ is a metric space and $\lambda>0$, we denote by $\lambda X$ the metric space $\left(X, \lambda \cdot d_{X}\right)$. The following lemma and its corollary show that the space $\bar{X}$ has a weak tangent space that contains $S_{2}$, see Chapters 7-8 of [7], and Chapter 9 of [9], for background on Gromov-Hausdorff convergence and weak tangent spaces.

Lemma 11.4. The slit carpet $S_{2}$ is the Gromov-Hausdorff limit of the sequence $\left(\bar{X}_{n}=2^{n} \pi_{\bar{X}}^{-1}\left(\left[0,1 / 2^{n}\right] \times\left[0,1 / 2^{n}\right]\right)\right)$.

Proof. We use Theorem 7.4 .12 from [7]. Let $\epsilon>0$ and $N \in \mathbb{N}$ be chosen so that $1 / 2^{N+1}<\epsilon$. The 1 -skeleton graph of the dyadic subdivision $\tilde{D}_{N}$ of $\bar{Q}_{0}$ into $2^{N+1} \times 2^{N+1}$ subsquares pulls back to a graph $D_{N}$ in $S_{2}$ via $\pi_{S_{2}}$ and graphs $D_{N, n}$ in each $\bar{X}_{n}$ via $\pi_{\bar{X}} \circ 2^{-n}$. This means that $D_{N}$ is a graph embedded in $S_{2}$ and $D_{N, n}$ is a graph embedded in $\bar{X}_{n}$ such that the sets of vertices are the sets of preimages of the vertices of $\tilde{D}_{N}$ by $\pi_{S_{2}}$ and $\pi_{\bar{X}} \circ 2^{-n}$ respectively. Two vertices are connected by an edge in $D_{N}$ or $D_{N, n}$ if and only if they are connected by an edge in $\tilde{D}_{N}$. Note that there are pairs of distinct vertices of $D_{N}$, respectively $D_{N, n}$, that get mapped to the same vertex of $\tilde{D}_{N}$. We do not connected such pairs of vertices by 
an edge. If $n \geq N$, the graphs $D_{N}$ and $D_{N, n}$ are identical. Since the vertices of these graphs form epsilon-nets, the lemma follows.

The following corollary is immediate:

Corollary 11.5. The completion of $X$ in the path metric has a weak tangent space that contains $S_{2}$.

A metric Sierpiński carpet $S$ is called porous if there exists $C \geq 1$ such that for every $p \in S$ and $0<r \leq \operatorname{diam}(S)$, there exists a peripheral circle $J$ in $S$ with $J \cap B(p, r) \neq \emptyset$ and

$$
\frac{r}{C} \leq \operatorname{diam}(J) \leq C \cdot r
$$

Lemma 11.6. The slit carpet $S_{2}$ cannot be quasisymmetrically embedded into the standard plane $\mathbb{R}^{2}$.

Proof. The metric Sierpiński carpet $S_{2}$ is porous, see Proposition 2.4 in [21], and its peripheral circles are uniform quasicircles, in fact they are isometric to circles. Assume that there is a quasisymmetric embedding $f: S_{2} \hookrightarrow \mathbb{R}^{2}$. An easy application of Proposition 10.8 in [14] implies that the image $f\left(S_{2}\right)$ is a porous metric Sierpiński carpet in the restriction of the Euclidean metric. The peripheral circles of $S_{2}$, and hence the boundaries of the complementary components of $f\left(S_{2}\right)$, are uniform quasicircles. Applying Theorem 8.2 to each complementary component of $f\left(S_{2}\right)$ now shows that $f\left(S_{2}\right)$ is porous as a subset of $\mathbb{R}^{2}$. Theorem 8.1 now states that the Assouad dimension, and hence the Hausdorff dimension, of $f\left(S_{2}\right)$ is strictly less than two. On the other hand, according Proposition 2.4 in [21], $S_{2}$ is Ahlfors 2-regular, and it contains a curve family of positive 2-modulus (see Lemma 4.2 in [21]). This contradicts Theorem 15.10 of [14].

Proof of Theorem 1.5. By Lemma 11.3, it suffices to show that $X$ cannot be quasisymmetrically embedded into the standard 2-sphere $\mathbb{S}^{2}$. Suppose such a quasisymmetric embedding $f: X \rightarrow \mathbb{S}^{2}$ can be found. It extends to a quasisymmetric embedding $\bar{f}$ of the completion $\bar{X}$ into $\mathbb{S}^{2}$ (Proposition 10.10 in [14]). The map $\bar{f}$ then induces a quasisymmetric embedding of every weak tangent space of $\bar{X}$ into the standard plane. Corollary 11.5 provides a weak tangent space of $\bar{X}$ that contains $S_{2}$. This contradicts Lemma 11.6.

\section{Open questions}

Question 12.1. In Theorem 1.4, can the assumption that $\partial X$ have finite rank be removed? It seems likely that this is the case. An affirmative answer is implied by an affirmative answer to the following question. Let $(X, d)$ be a metric space, 
homeomorphic to a domain in $\mathbb{S}^{2}$, that satisfies conditions (1)-(5) of Theorem 1.4, and has no isolated trivial boundary components. Consider the glued space $\left(Z, d_{Z}\right)$ formed from $\bar{X}$ and a collection of quasidisks $\left\{D_{i}\right\}$ corresponding to (all) the nontrivial components of $\partial X$, as in the proof of Theorem 1.4. Is it true that $\left(Z, d_{Z}\right)$ is homeomorphic to $\mathbb{S}^{2}$ ?

Question 12.2. Suppose that $(X, d)$ is a metric space, homeomorphic to a domain in $\mathbb{S}^{2}$, that satisfies the ALLC condition. Are the components of $\partial X$ uniformly relatively separated, quantitatively? Using the techniques of Section 4, it can be shown that the answer is "yes" in the case that $(X, d)$ is a domain in $\mathbb{S}^{2}$.

Question 12.3. Is there a quantitative statement, analogous to Theorem 1.4, that uniformizes onto the class of all circle domains? Given a particular circle domain $\Omega$ that does not have uniformly relatively separated boundary components, can one give sufficient intrinsic conditions for a metric space to be quasisymmetrically equivalent to $\Omega$ ?

\section{References}

[1] Bonk, M.: Quasiconformal geometry of fractals. In International Congress of Mathematicians. Vol. II, 1349-1373. Eur. Math. Soc., Zürich, 2006.

[2] Bonk, M.: Uniformization of Sierpiński carpets in the plane. Invent. Math. 186 (2011), no. 3, 559-665.

[3] Bonk, M., Heinonen, J. and Rohde, S.: Doubling conformal densities. J. Reine Angew. Math. 541 (2001), 117-141.

[4] Bonk, M., Heinonen, J. and Saksman, E.: The quasiconformal Jacobian problem. In In the tradition of Ahlfors and Bers, III, 77-96. Contemp. Math. 355, Amer. Math. Soc., Providence, RI, 2004.

[5] Bonk, M. And Kleiner, B.: Quasisymmetric parametrizations of two-dimensional metric spheres. Invent. Math. 150 (2002), no. 1, 127-183.

[6] Meyer, D.: Invariant Peano curves of expanding Thurston maps. Acta Math. 210 (2013), no. 1, 95-171.

[7] Burago, D., Burago, Y., And Ivanov S.: A course in metric geometry. Graduate Studies in Mathematics 33, American Mathematical Society, Providence, RI, 2001.

[8] Daverman, R.: Decompositions of manifolds. Pure and Applied Mathematics 124, Academic Press, Orlando, FL, 1986.

[9] David, G. And Semmes, S.: Fractured fractals and broken dreams. Oxford Lecture Series in Mathematics and its Applications 7, The Clarendon Press Oxford University Press, New York, 1997.

[10] Goluzin, G.: Geometric theory of functions of a complex variable. Translations of Mathematical Monographs 26, Amer. Math. Soc., Providence, RI, 1969.

[11] Grötzsch, H.: Über das Parallelschlitztheorem der konformen Abbildung schlichter Bereiche. Berichte Leipzig 84 (1932), 15-36.

[12] Haïssinsky, P.: A sewing problem in metric spaces. Ann. Acad. Sci. Fenn. Math. 34 (2009), no. 2, 319-345. 
[13] He, Z. and Schramm, O.: Fixed points, Koebe uniformization and circle packings. Ann. of Math. (2) 137 (1993), no. 2, 369-406.

[14] Heinonen, J.: Lectures on analysis on metric spaces. Universitext, Springer-Verlag, New York, 2001.

[15] Heinonen J. and Koskela, P.: Quasiconformal maps in metric spaces with controlled geometry. Acta Math. 181 (1998), no. 1, 1-61.

[16] Herron D. and Meyer D.: Quasicircles and bounded turning circles modulo biLipschitz maps. Rev. Mat. Iberoam. 28 (2012), no. 3, 603-630.

[17] Hurewicz, W. and Wallman, H.: Dimension Theory. Princeton Mathematical Series 4, Princeton University Press, Princeton, NJ, 1941.

[18] Kapovich, M. And Kleiner, B.: Hyperbolic groups with low-dimensional boundary. Ann. Sci. École Norm. Sup. (4), 33 (2000), no. 5, 647-669.

[19] Koebe, P.: Über die Uniformisierung der algebraischen Kurven. I. Math. Ann. 67 (1909), no. 2, 145-224.

[20] Koebe, P.: Abhandlungen zur Theorie der konformen Abbildung. Math. Z. 7 (1920), no. 1-4, 235-301.

[21] Merenkov, S.: A Sierpiński carpet with the co-Hopfian property. Invent. Math. 180 (2010), no. 2, 361-388.

[22] Meyer, D.: Bounded turning circles are weak-quasicircles. Proc. Amer. Math. Soc. 139 (2011), no. 5, 1751-1761.

[23] Moore, R.: Concerning upper semicontinuous collections of continua. Trans. Amer. Math. Soc. 27 (1925), no. 4, 416-428.

[24] Munkres, J.: Topology: a first course. Prentice-Hall, Englewood Cliffs, NJ, 1975.

[25] Tukia, P. And VÄısÄlä, J.: Quasisymmetric embeddings of metric spaces. Ann. Acad. Sci. Fenn. Ser. A I Math. 5 (1980), no. 1, 97-114.

[26] Wilder, R.: Concerning simple continuous curves and related point sets. Amer. J. Math. 53 (1931), no. 1, 39-55.

[27] Wildrick, K.: Quasisymmetric parametrizations of two-dimensional metric planes. Proc. Lond. Math. Soc. (3) 97 (2008), no. 3, 783-812.

[28] Wildrick, K.: Quasisymmetric structures on surfaces. Trans. Amer. Math. Soc. 362 (2010), no. 2, 623-659.

[29] Zippin, L.: A characterisation of the closed 2-cell. Amer. J. Math. 55 (1933), no. 1-4, $207-217$.

Received November 21, 2011.

Sergei Merenkov: Department of Mathematics, University of Illinois at UrbanaChampaign, 1409 W Green Street, Urbana, IL 61801, USA.

E-mail: merenkov@illinois.edu

Kevin Wildrick: Mathematisches Institut, Universität Bern, Sidlerstrasse 5, 3012 Bern, Switzerland.

E-mail: kevin.wildrick@math.unibe.ch

S. M. was supported by NSF grants DMS-0653439 and DMS-1001144. K. W. was supported by Academy of Finland grants 120972 and 128144 . 